\title{
A Novel Cooperation-Based Network Coding Scheme for Walking Scenarios in WBANs
}

\author{
Hongyun Zhang, ${ }^{1,2}$ Farzad Safaei, ${ }^{1}$ and Le Chung Tran ${ }^{1}$ \\ ${ }^{1}$ School of Electrical, Computer and Telecommunications Engineering, Faculty of Engineering and Information Science, \\ University of Wollongong, Wollongong, NSW 2522, Australia \\ ${ }^{2}$ College of Computer, National University of Defense Technology, Changsha, Hunan 410073, China
}

Correspondence should be addressed to Hongyun Zhang; hz697@uowmail.edu.au

Received 29 May 2017; Revised 30 July 2017; Accepted 6 August 2017; Published 14 September 2017

Academic Editor: Donatella Darsena

\begin{abstract}
Copyright (C) 2017 Hongyun Zhang et al. This is an open access article distributed under the Creative Commons Attribution License, which permits unrestricted use, distribution, and reproduction in any medium, provided the original work is properly cited.

In Wireless Body Area Networks (WBANs), the tradeoff between network throughput and energy efficiency remains a key challenge. Most current transmission schemes try to cope with the challenge from the perspective of general Wireless Sensor Networks (WSNs), which may not take the peculiarities of WBAN channels into account. In this paper, we take advantage of the correlation of on-body channels in walking scenarios to achieve a better tradeoff between throughput and energy consumption. We first analyze the characteristics of on-body channels based on realistic channel gain datasets, which are collected by our customized wireless transceivers in walking scenarios. The analytical results confirm the rationale of our newly proposed transmission scheme A3NC, which explores the combination of the aggregative allocation (AA) mechanism in MAC layer and the Analog Network Coding (ANC) technique in PHY layer. Both theoretical analyses and simulation results show that the A3NC scheme achieves significant improvement in upload throughput and energy efficiency, compared to the conventional approaches.
\end{abstract}

\section{Introduction}

In recent years, with the technological advancements in wireless communication, Microelectromechanical Systems (MEMS), and integrated circuits, WBANs have become a more practical and promising technology [1]. However, WBANs have strict energy constraints as frequent change of batteries is inconvenient or even infeasible. Besides, sensory devices are usually placed either in or on the human body; thus the maximum radiated transmission power is restricted to comply with the limitation of Specific Absorption Rate (SAR) of local regulatory bodies (the limitation of Federal Communications Commission is $1.6 \mathrm{~W} / \mathrm{Kg}$ ). Meanwhile, up to $10 \mathrm{Mbps}$ throughput should be offered to satisfy the required set of entertainment and healthcare services [2]. It is a challenging task to maintain a high throughput while fulfilling the specific energy efficiency requirement of WBANs.

Propagation paths in WBANs can experience fading due to different reasons, such as energy absorption, reflection, diffraction, shadowing by the body, and body postures [3]. All these unique features lead to high packet losses. Relay nodes, if deployed outside of the human body, might possess better channels and less stringent energy limitations. Therefore, cooperative communication [4] has received considerable interest in recent years [5-8]. Initial solutions apply the existing methods, which were proposed for general Wireless Sensor Networks (WSNs), to WBANs. These schemes may not be optimized for the unique challenges and specific characteristics of WBANs, such as spatial correlation of channels, stringent tradeoff between energy requirement and throughput gains, and Quality of Service (QoS) requirements.

Due to the broadcast nature of wireless networks, network coding (NC) [9], which enables nodes to code or mix packets (or symbols) before forwarding, is a potential method to produce a significant improvement in the throughput, reliability, manageability, and QoS of wireless networks [10, 11]. The applications of network coding in WBANs are still an emerging area and most existing works consider the expansions of conventional wireless network coding schemes. The works of [12-15] expanded the cluster-based scheme 
proposed in [16], which divides intermediate relay nodes into multiple clusters, and NC packets are transmitted between clusters. However, because only one-hop or two-hop star topologies are considered suitable for most WBAN systems [2], the feasibility of cluster-based schemes is limited. In [17], the authors proposed practical NC implementations in the context of WBANs. The linear NC is performed in the source node to improve the energy efficiency. In [18], the relay first demodulates the received symbols from sensor nodes and then generates a network coded symbol by the XOR operation of selected symbols. In a similar way, the works in [19-22] also utilize the NC technology in the relay node to improve the transmission efficiency. The authors of [23] focus on the energy efficiency of cooperation transmission; decode-andforward network coding (DF-NC) is proposed to aid relay cooperative transmission. The DF-NC scheme achieves a near optimal outage probability performance while maximizing the energy efficiency of WBANs by fixing the average number of transmissions per node. In [24], the authors explored the application of NC for the cloud-assisted ambient assisted living (AAL) environments. Random linear network coding (RLNC) technology is employed to the source node to improve the efficiency of retransmission. Some other works provided new perspectives to the harnessing of $\mathrm{NC}$ in WBANs. In [25], the cooperative compressed sensing approach is combined with RLNC technology to increase the energy efficiency of WBANs. However, most of the previous works did not consider the particular characteristics of WBAN channel when employing the NC technology.

One particular feature of WBANs is that the signal attenuation is significantly affected by the shadowing of body tissues in addition to the distance between two devices. In other words, the movement and posture of the human body have a dramatic effect on the strength of the received signal. Motivated by the spatial cross-correlation of channels in walking scenarios [26, 27], we proposed a novel NC transmission scheme, named A3NC, in our previous work [28]. However, the simulation model in [28] was built based on a distance-based channel model, rather than adopting the realistic channel gain. Also, the theoretical performance analysis was not provided in [28]. Moreover, the cross-correlation of on-body channels was presumed to be significant in [28]. In [29], we performed a full-scale experiment-based analysis of on-body channels in the walking scenarios and found that the on-body channels do not manifest meaningful crosscorrelation, either negative or positive. The experimental measurements presented later in this paper show that the path loss discrepancy between the on-body channels, rather than the cross-correlation, is significant, and it provides a more suitable basis for the new proposed scheme A3NC. However, [29] did not provide any analyses or evaluation of our newly proposed A3NC scheme. In this paper, we will derive indepth mathematical analyses and performance evaluations, based on real path loss datasets.

The contributions of this paper are as follows:

(i) The real channel gain datasets in walking scenarios are collected by our customized portable wireless transceiver. In-depth analyses of these experimental datasets confirm the basis of our newly proposed A3NC transmission scheme.

(ii) Instead of the distance-based channel model used in [28], the realistic channel gain datasets collected from our measurement campaign are imported into the IEEE 802.15.6-compatible simulation model to evaluate the performance of A3NC. Simulations show that the proposed $\mathrm{A} 3 \mathrm{NC}$ achieves a better performance from upload throughput, energy efficiency, and throughput balance perspectives.

(iii) To deepen the understanding of all key parameters that affect system performance, we provide the mathematical analyses from all three perspectives, namely, upload throughput, energy efficiency, and throughput balance.

The rest of the paper is organized as follows. In Section 2, the system model is presented. Then, the characteristics of on-body channels in the walking scenarios are analyzed in Section 3. Section 4 details the proposed A3NC scheme. In Section 5, the mathematical analyses are provided. Performance evaluation results based on the realistic channel gain datasets are presented in Section 6. Finally, Section 7 concludes the paper.

\section{System Model}

In this section, we first present the network model for typical walking scenarios. Then channel models, including the onbody channel and relay-related channel, are introduced. Finally, the notations utilized throughout this article are presented.

2.1. Network Model. We consider the walking scenarios where two sensors $\left(\mathrm{SN}_{1}\right.$ and $\left.\mathrm{SN}_{2}\right)$ mounted on the wrists communicate with a hub, and a relay node deployed outside the human body is optional. As the hub is expected to be larger than a sensor node, the torso is considered to be a preferable part to mount the hub. In this paper, we consider two typical device deployments as depicted in Figure 1, where the hub is placed on the abdomen (attached to the belt buckle) or on the back collar. The main task for the sensors is to continually upload monitoring data to the hub with or without the help of the relay node. All wireless devices operate in the half-duplex mode, and Time Division Multiple Access (TDMA) is used to schedule the channel resource.

2.2. Channel Model. Some current works, including our paper [28], simply utilize the distance-based formula to quantify the strength of received signal power. However, the path loss of on-body channels in WBANs is affected by many factors, such as the absorption effect of human tissues and the mobility of the human body. In this work, the wireless channels in the walking scenarios are classified into two categories: on-body channel and relay channel (as shown in Figure 2). Since only the sensors and the hub are deployed on the body, the channel between the sensors and the hub is named as on-body channel. On the other hand, the channel to or from the relay node is called relay channel. 


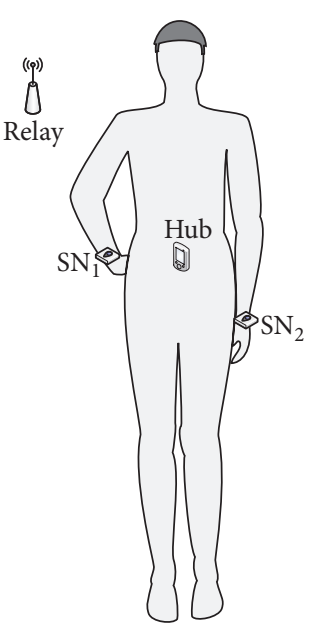

(a)

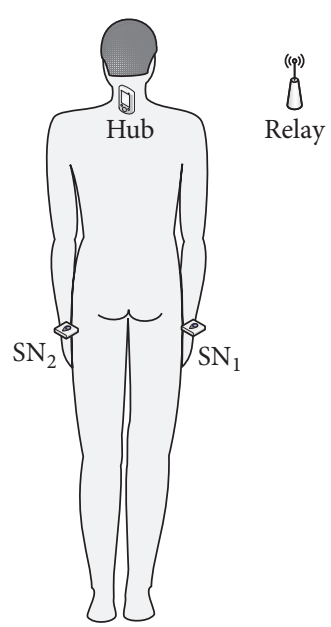

(b)

Figure 1: Deployment of the sensors, relay, and hub.

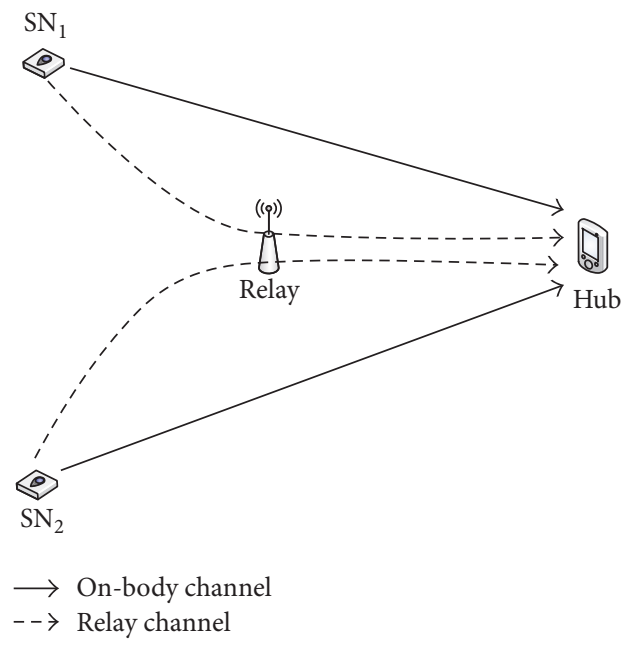

FIgURE 2: The on-body channel and relay channel.

2.2.1. On-Body Channel Model. Due to the high variability of on-body channels, neither distance-based nor other formulabased methods seem to be sufficient to describe the onbody channel condition, especially in the activity scenarios. Therefore, adopting channel gain datasets collected from the real walking scenarios to model the on-body channel is a better choice. To collect the realistic on-body channel gain data, we constructed wireless transceivers, as illustrated in Figure 3. Each wireless transceiver consists of one radio module, one microcontroller, and one MicroSD card. The main function of the transceivers is to transmit/receive continuous data packets to/from each other, thus facilitating the analysis of channel gains. Every testbed is composed of three printed circuit boards, and they stack on each other to make the system self-contained. More details about the hardware can be found in [29].

As demonstrated in [30,31], in narrowband communication environments, the on-body channels show prominent reciprocity, which means the channel profiles of downlink and uplink are around the same. Therefore, the channel gain can be estimated by the measurement of downlinks (from the hub to the sensors). The transmitter attached on the abdomen or the back collar continuously broadcasts sample packets to the receivers located on the two wrists, and the receivers record the channel gain data by time sequence. These datasets created from the experiment are utilized to model the onbody channels. More details about these experiments and the analysis of channel gain datasets will be presented in the next section.

2.2.2. Relay Channel Model. In the context of WBANs, due to severe fading caused by body shadowing, the relay node may improve the transmission reliability effectively. As the off-body channel is considered to be more stable than the on-body channel, we assume the relay node is deployed outside the human body. In this work, the channel $\mathrm{SN}_{i}$-relay and channel relay-hub are collectively called "relay channel." Besides, since the swing motions of two arm are symmetrical when the human is walking, the average packet delivery ratios (PDRs) tend to be the same for the two relay channels, that is, $\mathrm{SN}_{1}$-relay-hub and $\mathrm{SN}_{2}$-relay-hub. Accordingly, without loss of generality, we assume the average PDRs of the two relay channels are the same.

2.3. Notations. $R$ (Kbps) refers to the transmission rate of the upload data from the sensor to the hub, which is assumed to be the same for the two sensors. $L_{p}$ (Kbits) denotes the length of one packet. $T_{c}$ is the total time assigned to the two sensors and the relay node (if exist). Both $T_{s}$ and $T_{c}$ are in unit of second. $\beta_{i}$ represents the average PDR of the channel $\mathrm{SN}_{i}$-hub and $\beta_{r}$ is the average PDR of the channel SN-relayhub. As mentioned before, the two relay channels are assumed to have the same $\beta_{r}$. In the proposed A3NC scheme, two sensors share a common time period to upload monitoring data. Therefore, some packets may be lost due to the signal collision, and we denote the average packet error ratio (PER) 


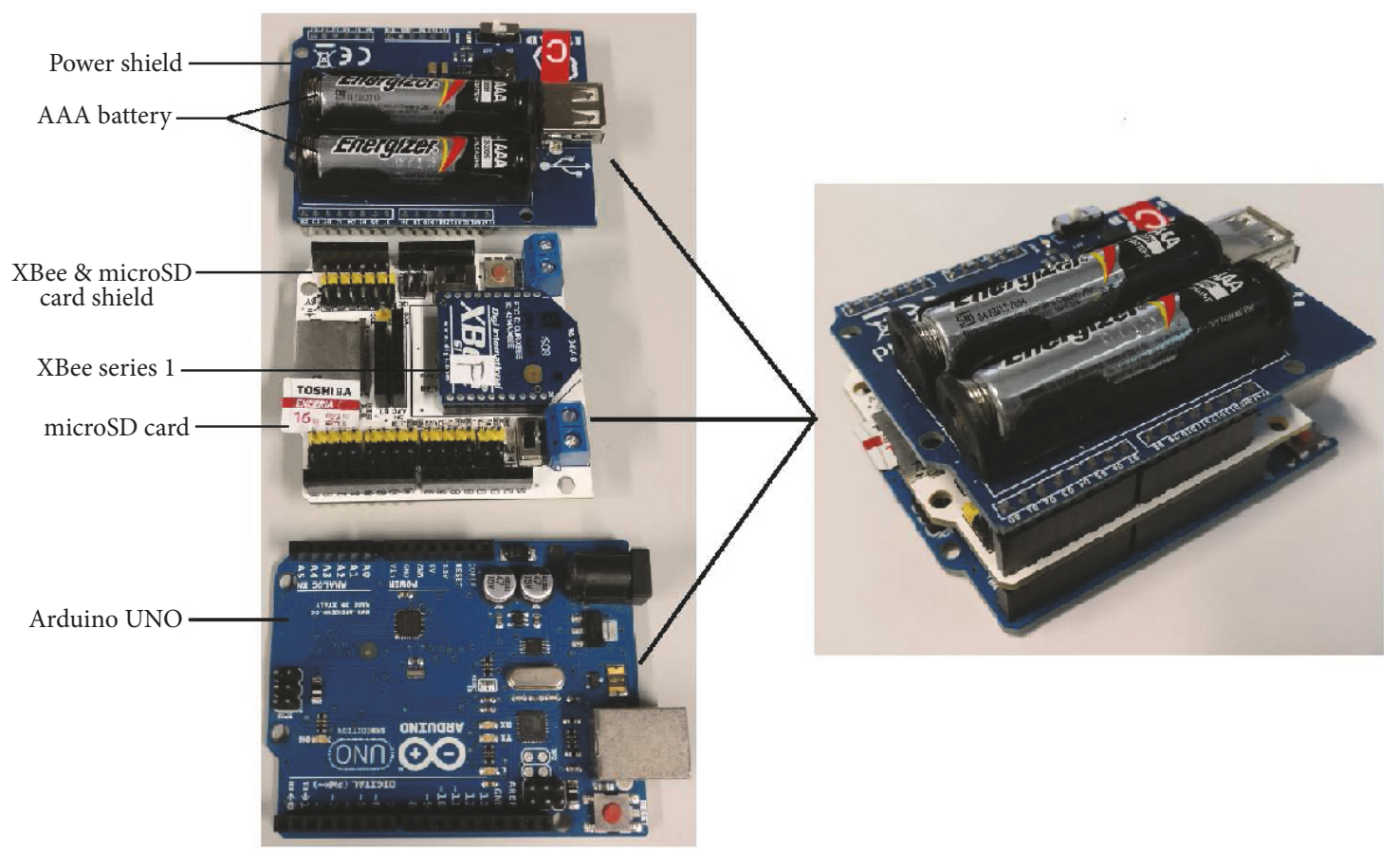

FIgURE 3: Components of the wireless transceiver.

caused by collision as $e$. Note that $\beta_{i}$ is measured when the channel is only occupied by $\mathrm{SN}_{i}$, without taking the packet losses caused by signal collisions into account. $U_{i}$ denotes the actual upload throughput from $\mathrm{SN}_{i}$ to the hub with the influence of fading and collision. $\mathscr{P}_{w}$ is the working power of the sensor node, and we assume the two sensors have the same working power.

\section{Characteristics of On-Body Channels}

In this section, we first present the measurement setups for typical walking scenarios. Then the characteristics of the onbody channels are analyzed based on the datasets collected from these measurements.

3.1. Measurement Setups. The measurements were conducted in both indoor and outdoor environments. The outdoor environment is an open oval field of about 13,000 square meters. The indoor environment is a hallway inside a building. Accordingly, there are four different walking scenarios generated based on the environment and the location of the hub. For the rest of the paper, four scenarios are named as "Indoor + Belt," "Indoor + Collar," "Outdoor + Belt," and "Outdoor + Collar." Specifically, the transmitter located on the belt buckle or the back collar continuously broadcasts packets to the two receivers bound on the wrists with the transmit (Tx) power of $0 \mathrm{dBm}$ and the sampling frequency of $200 \mathrm{~Hz}$. Then the receivers record the RSSI (Received Signal Strength Indicator) value of received packets, the timestamp, and the packet sequence number in the MicroSD card. Note that, as the Tx power is $0 \mathrm{dBm}$, the inverse of the RSSI is the path loss.
To explore the effect of the antenna direction and the shadowing effect of wrists, the portable transceivers are rolled around the wrist in four directions: $0^{\circ}, 90^{\circ}, 180^{\circ}$, and $270^{\circ}$, as depicted in Figure 4. Consequently, there are four different measurement setups (or subscenarios) in each scenario.

3.2. Channel Data Statistics. We first analyze the RSSI data for four different scenarios. Figures 5-8 present typical timevarying RSSI data for the two links from the hub to the two sensors. $\mathrm{RSSI}_{1}$ and $\mathrm{RSSI}_{2}$ refer to the RSSI of the packets received by $\mathrm{SN}_{1}$ and $\mathrm{SN}_{2}$, respectively. Observations that can be derived from the four figures are listed below.

(i) When the hub is on the "Collar," the channel path losses are much bigger than the "Belt" cases. The main reason is that, in the "Collar" cases, the signal is affected by not only the shadowing of the torso but also the shadowing of the upper limbs

(ii) The comparison between the indoor and outdoor environments shows that there exist more small-scale fluctuations in the indoor environment, resulting from the reflection from surrounding objects, such as desks, wall, and roof.

(iii) The direction of transceivers also affects the signal attenuation. When the hub is on the abdomen, the $180^{\circ}$ cases experience the biggest path loss. The reason is that when the transceivers are bound on the top of the wrists, the signal is affected by not only the shadowing of the torso but also the shadowing of the wrists themselves. Similarly, in the "Collar" cases, both $90^{\circ}$ and $180^{\circ}$ cases bring more shadowing from the upper limbs, leading to lower RSSI values. 


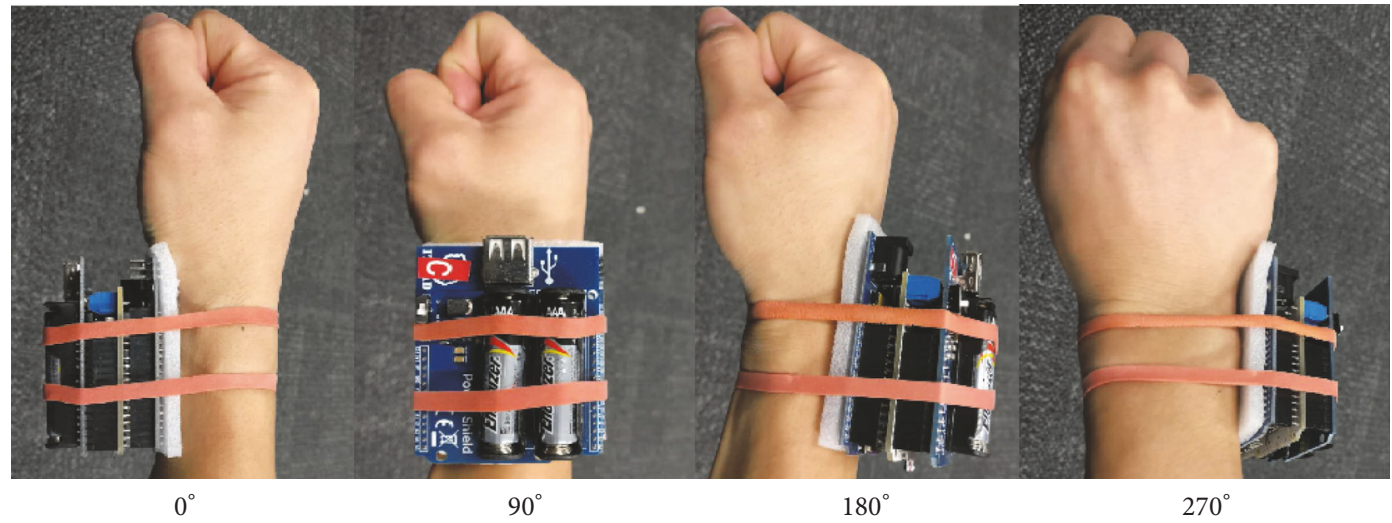

FIgURE 4: Four directions of the transceiver on the wrist.

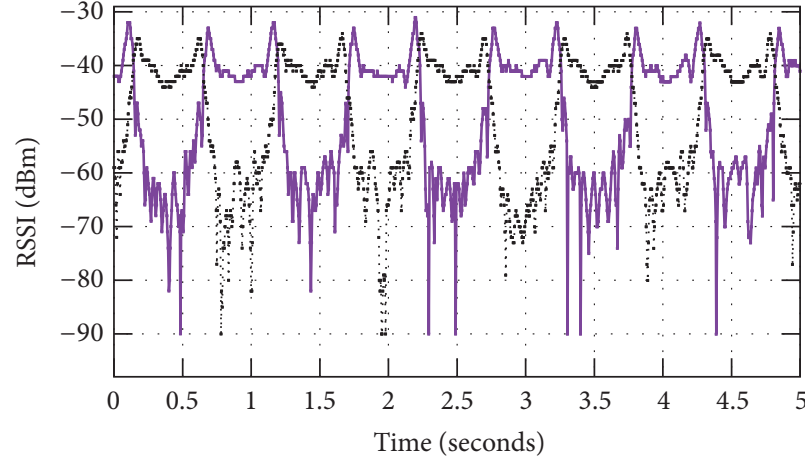

- $\mathrm{RSSI}_{1}$

…... RSSI $_{2}$

(a) Indoor + Belt, $0^{\circ}$

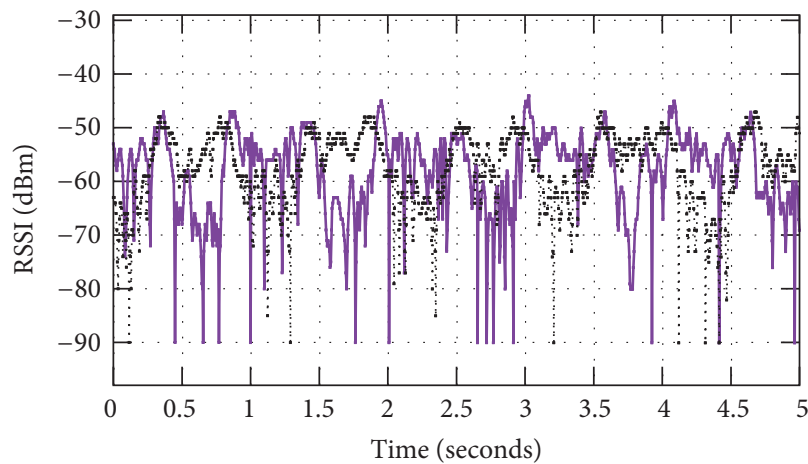

$-\mathrm{RSSI}_{1}$

…... $\mathrm{RSSI}_{2}$

(c) Indoor + Belt, $180^{\circ}$

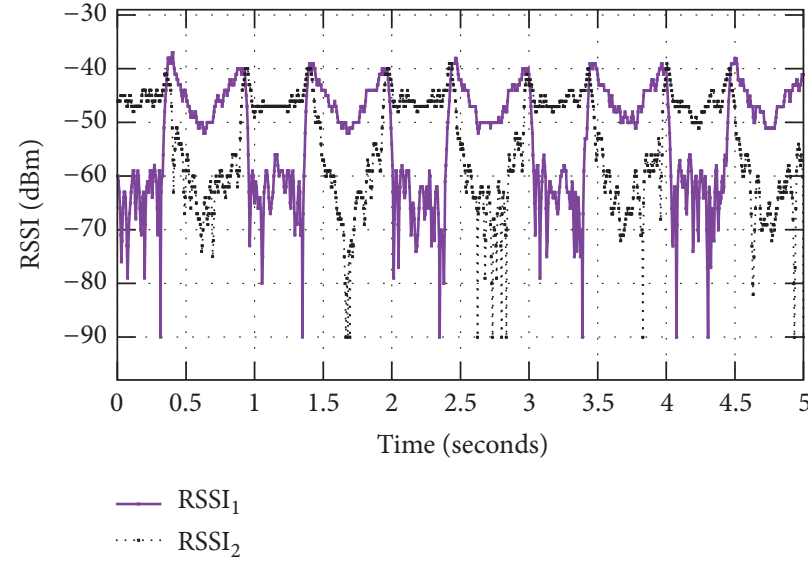

(b) Indoor + Belt, $90^{\circ}$

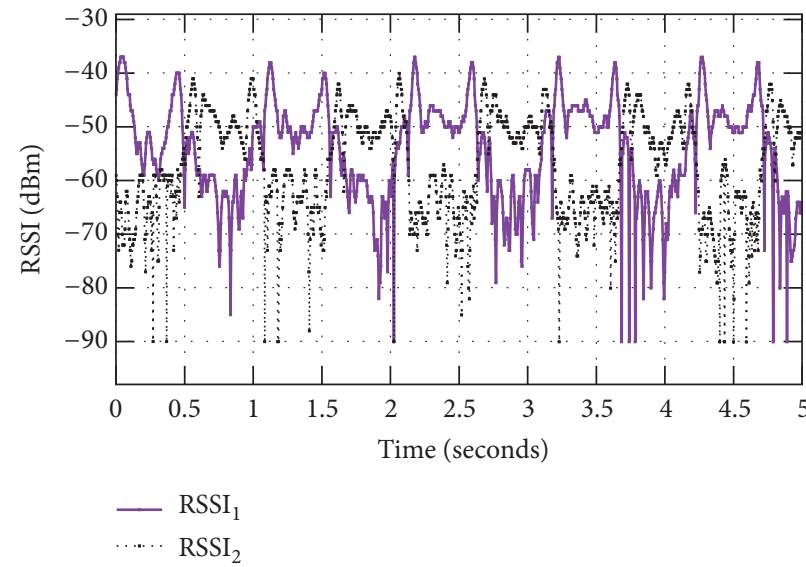

(d) Indoor + Belt, $270^{\circ}$

FIGURE 5: RSSI versus time in the "Indoor + Belt" scenario.

Next, we analyze the cross-correlation between the two links from the sensors to the hub. The Pearson productmoment correlation coefficient (PCC) [32] is adopted to evaluate the cross-correlation.

$$
\rho_{c}=\frac{\sum_{n=1}^{N}(x(n)-\bar{x})(y(n)-\bar{y})}{\sqrt{\sum_{n=1}^{N}(x(n)-\bar{x})^{2}} \sqrt{\sum_{n=1}^{N}(y(n)-\bar{y})^{2}}},
$$

where $\rho_{c}$ is the correlation coefficient between $x(n)$ and $y(n)$; $\bar{x}$ and $\bar{y}$ are the mean path loss values of the link $\mathrm{SN}_{1}$-hub and $\mathrm{SN}_{2}$-hub, respectively. $N=12,000$ is the number of samples in each measurement.

Table 1 presents the correlation coefficient for different experimental setups. It is found that the walking scenarios exhibit relatively small spatial cross-correlation coefficients, 


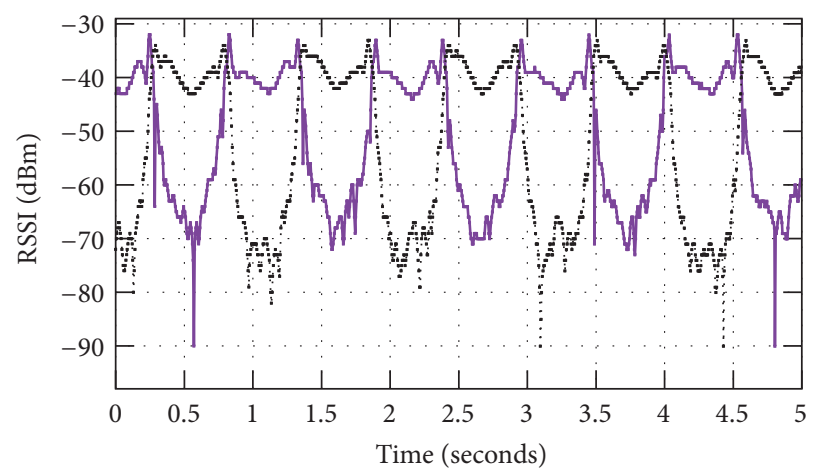

- $\mathrm{RSSI}_{1}$

…... RSSI $_{2}$

(a) Outdoor + Belt, $0^{\circ}$

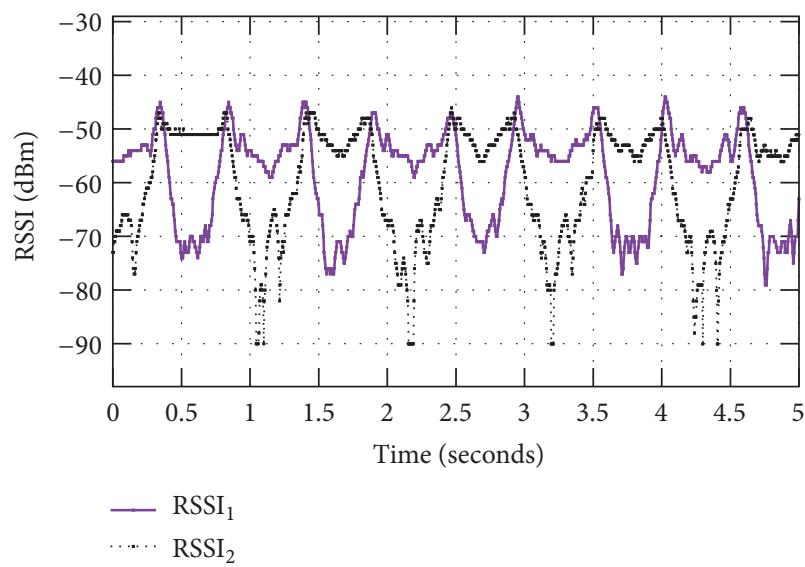

(c) Outdoor + Belt, $180^{\circ}$

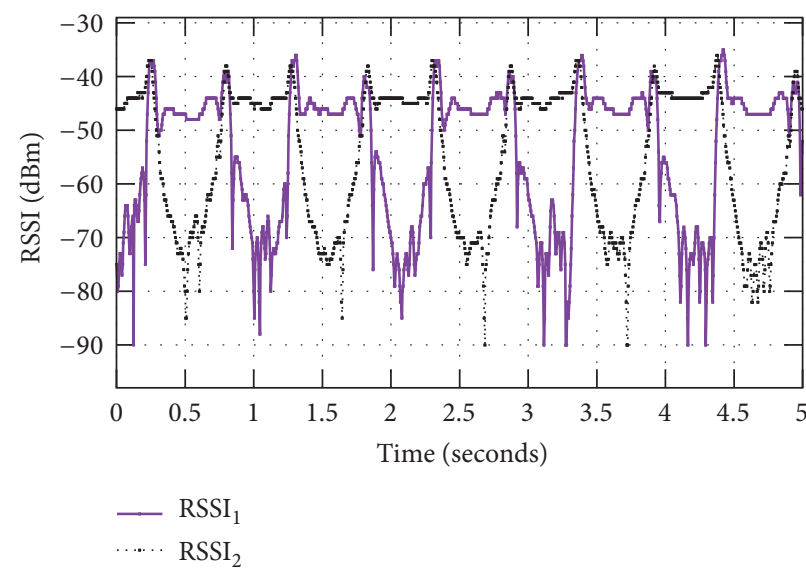

(b) Outdoor + Belt, $90^{\circ}$

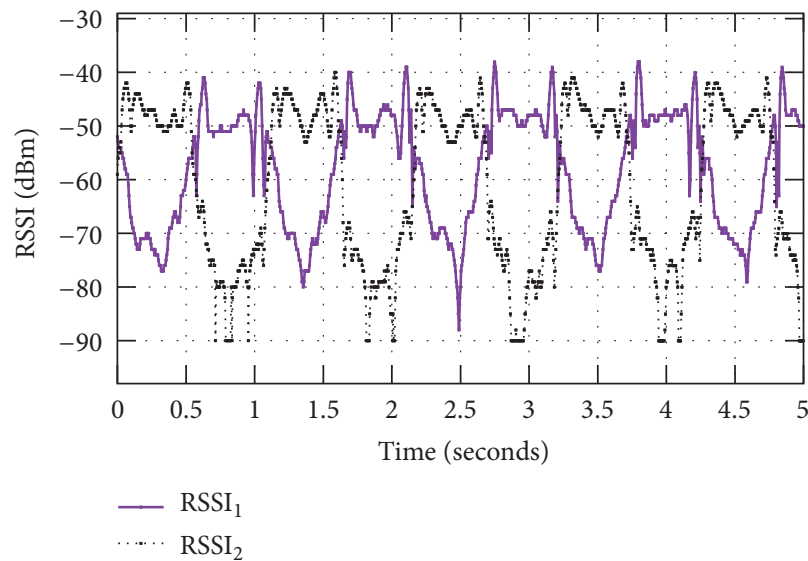

(d) Outdoor + Belt, $270^{\circ}$

FIGURE 6: RSSI versus time in the "Outdoor + Belt" scenario.

TABLE 1: Cross-correlation between two links.

\begin{tabular}{lcccc}
\hline & \multicolumn{2}{c}{ Indoor } & \multicolumn{2}{c}{ Outdoor } \\
& Belt & Collar & Belt & Collar \\
\hline $0^{\circ}$ & -0.45 & -0.08 & -0.42 & -0.27 \\
$90^{\circ}$ & 0.15 & 0.00 & 0.43 & 0.14 \\
$180^{\circ}$ & 0.20 & 0.06 & 0.22 & 0.09 \\
$270^{\circ}$ & -0.27 & -0.26 & -0.30 & -0.44 \\
\hline
\end{tabular}

as the spatial cross-correlation is generally considered to be significant when its absolute value is 0.7 or greater. Further, the cross-correlations vary dramatically with the network deployment, including the placement of the hub and sensors, surrounding environment, and the direction of transceivers. When the radio device rolls around the wrist, that is, $0^{\circ}, 90^{\circ}$, $180^{\circ}$, and $270^{\circ}$, the PCC also varies accordingly. The differences mainly result from the change of antenna direction and the change of shadowing from the arms. Especially for the "Collar" cases, the significant shadowing coming from both torso and upper limbs outweighs other effects, leading to a relatively insignificant PCC.

The cross-correlation between different links is considered to be an instructive parameter for the design of communication systems. However, a small absolute value of PCC does not necessarily mean that two links are entirely independent. Take the subscenario of Figure 6(c) as an example. The PCC of the two channels is 0.22 , but the two channels are obviously not independent. In this paper, we focus on a simple but important parameter, namely, the path loss discrepancy (in $\mathrm{dB}$ ), referred to as PLD, to reflect the actual difference between two channel gains.

As shown in Figure 9, the probability distributions of the PLD are different for the four scenarios, but they all show a high proportion for the case when the PLD is greater than $5 \mathrm{~dB}$. Besides, the PLD medians for four experimental setups from Figures 9(a) -9 (d) are $15 \mathrm{~dB}, 7 \mathrm{~dB}, 21 \mathrm{~dB}$, and $11 \mathrm{~dB}$, respectively. In the outdoor environment, the PLD tends to be greater than that in the indoor environment. The main reason is that the reflections from surrounding objects in the indoor environment narrow the gap between the RSSI of the two channels. Besides, the "belt" cases experience a bigger PLD than the "collar" cases, mainly because the shadowing effect is stronger in the latter.

Assuming that the environmental noise is relatively small, the PLD is the major constituent of the overall SNR (Signalto-Noise Ratio). Meanwhile, SNR is a crucial parameter for the bit error ratio (BER). The relationship between SNR 


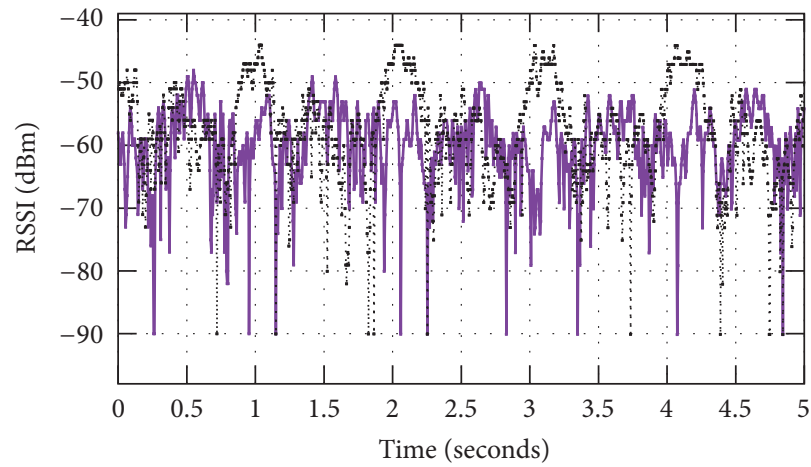

-. $\mathrm{RSSI}_{1}$

(a) Indoor + Collar, $0^{\circ}$

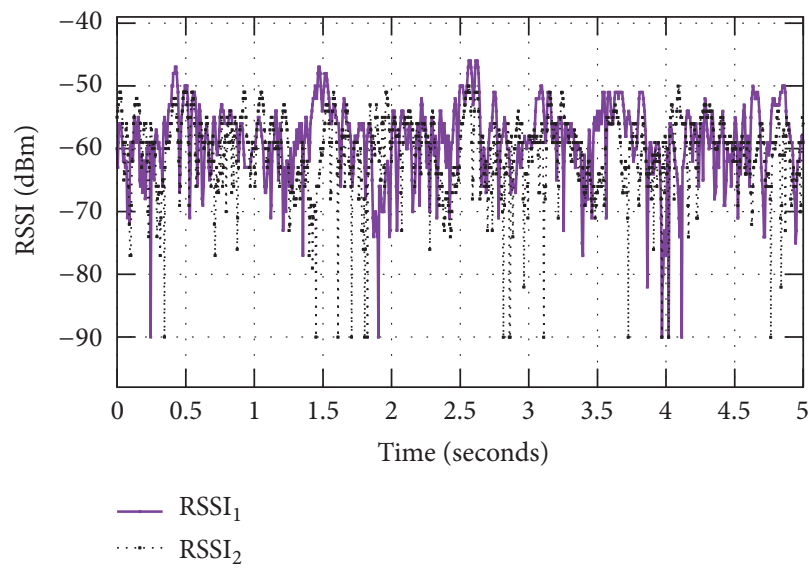

(c) Indoor + Collar, $180^{\circ}$

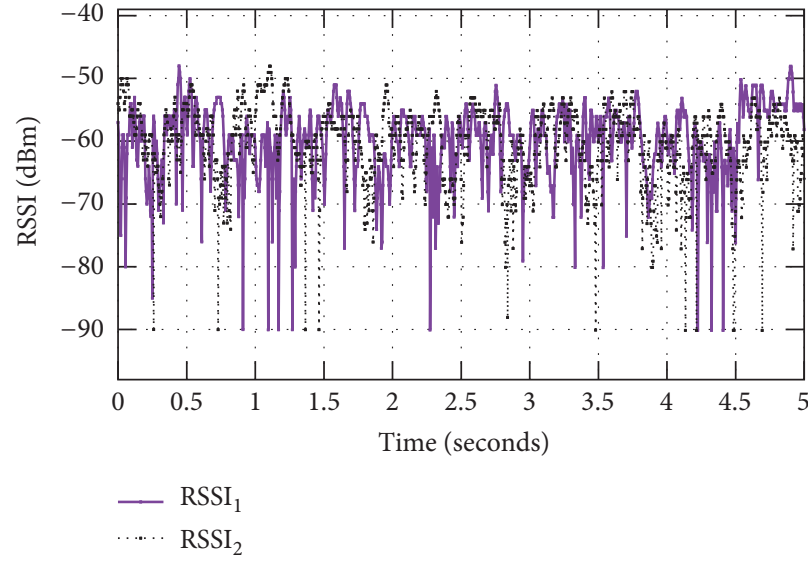

(b) Indoor + Collar, $90^{\circ}$

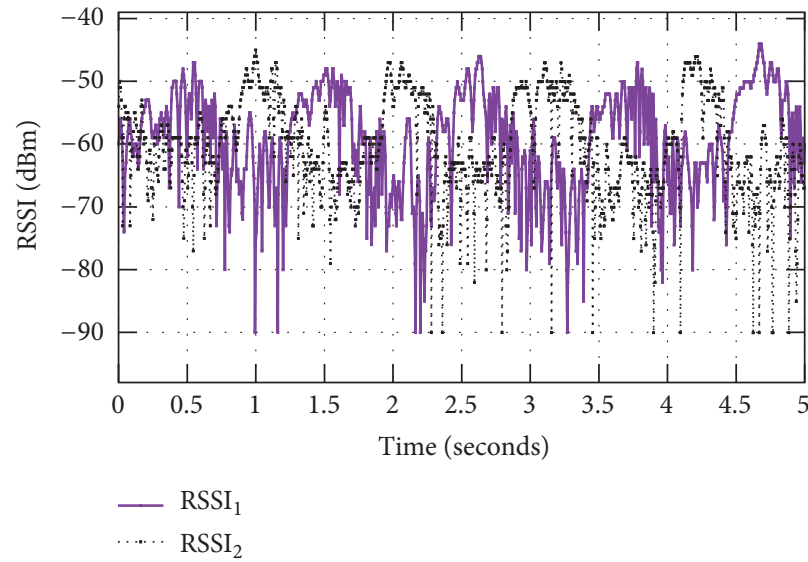

(d) Indoor + Collar, $270^{\circ}$

Figure 7: RSSI versus time in the "Indoor + Collar" scenario.

and BER has been investigated intensively in the literature $[33,34]$. When SNR $>7 \mathrm{~dB}$, the system achieves a BER lower than $1 \times 10^{-2}$ for the most low-order modulation schemes, which may be considered to be sufficient for practical implementation. Accordingly, if two sensors located on the wrists concurrently broadcast packets to the hub on the torso in walking scenarios, the hub can restore at least one signal with a high probability. In other words, although the two sensors transmit simultaneously, the probability of packet collisions is small. Consequently, the observed PLD in walking scenarios may be sufficient to justify the rationale for simultaneous transmission from the two sensors to the hub, which is the core idea of our proposed A3NC scheme. The A3NC scheme will be detailed in the next section. More accurate quantitative mathematical analyses of this proposed scheme will be provided in Section 5 .

\section{Joint Analog Network Coding and Channel Allocation}

In A3NC, the aggregative allocation (AA) mechanism and the ANC [35] technique are incorporated to improve the system throughput and energy efficiency in walking scenarios. AA is the implementation method of simultaneous transmission in IEEE 802.15.6. ANC technique mainly focuses on the problem of energy efficiency, which is a crucial performance metric for WBANs. These two components of the proposed A3NC scheme are described below.

4.1. Aggregative Allocation. As mentioned in the previous section, the significant PLD in the walking scenarios is the motivation for simultaneous transmissions of different source nodes. The proposed AA mechanism aims to make the simultaneous transmission compatible with the IEEE 802.15.6 standard. Therefore, it is instructive to introduce the structure of the IEEE 802.15.6 superframe briefly. As depicted in Figure 10, a superframe is divided into Exclusive Access Phases (i.e., EAP1 and EAP2), Random Access Phases (i.e., RAP1 and RAP2), Managed Access Phases (MAP), and a Contention Access Phase (CAP) [2]. In EAP, RAP, and CAP periods, nodes contend for the channel allocation using either CSMA/CA (carrier sense multiple access with collision avoidance) or slotted Aloha access procedure. In MAP periods, scheduled, unscheduled, and improvised access methods are possible. The scheduled access procedure is generally used for applications that involve periodic monitoring. 


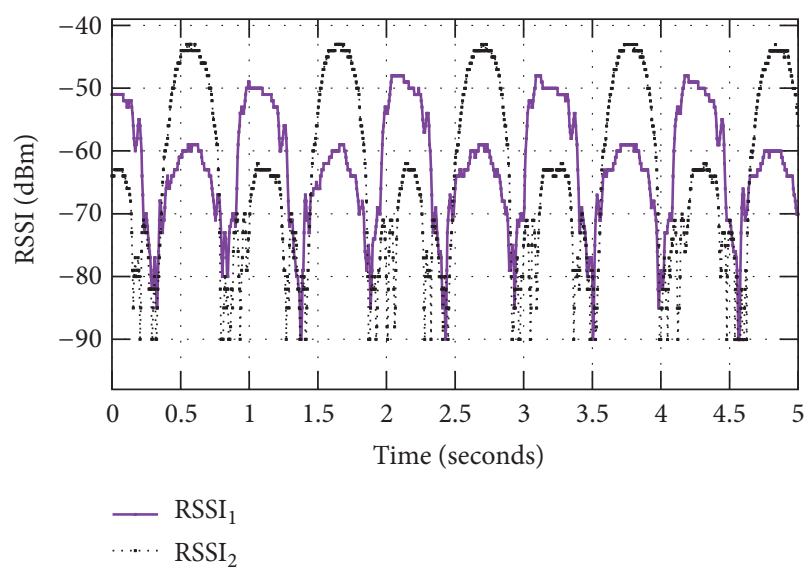

(a) Outdoor + Collar, $0^{\circ}$

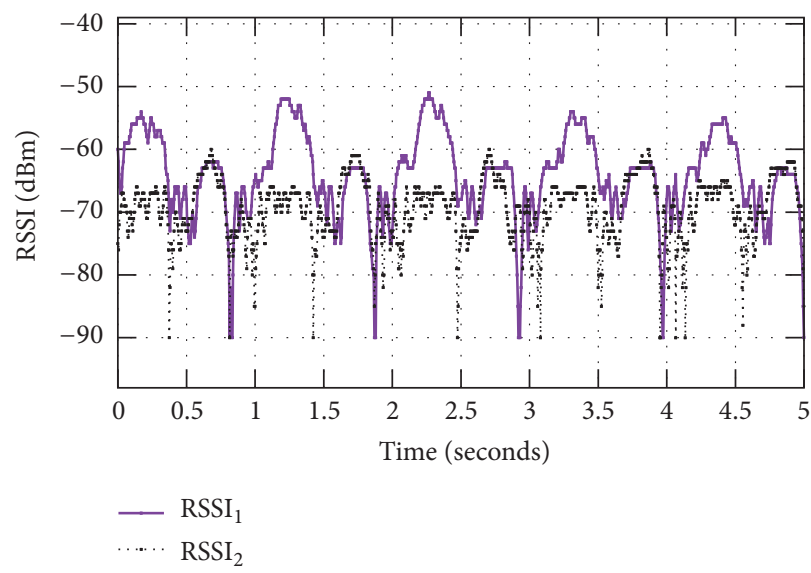

(c) Outdoor + Collar, $180^{\circ}$

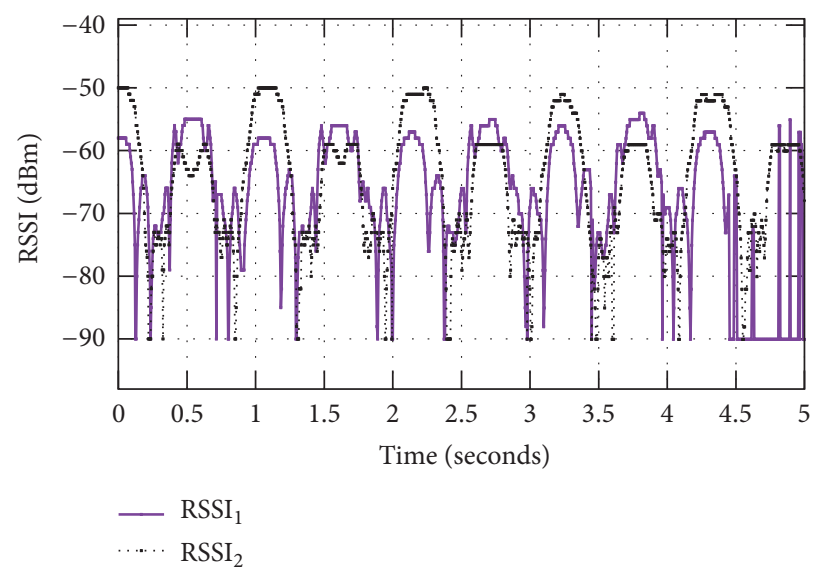

(b) Outdoor + Collar, $90^{\circ}$

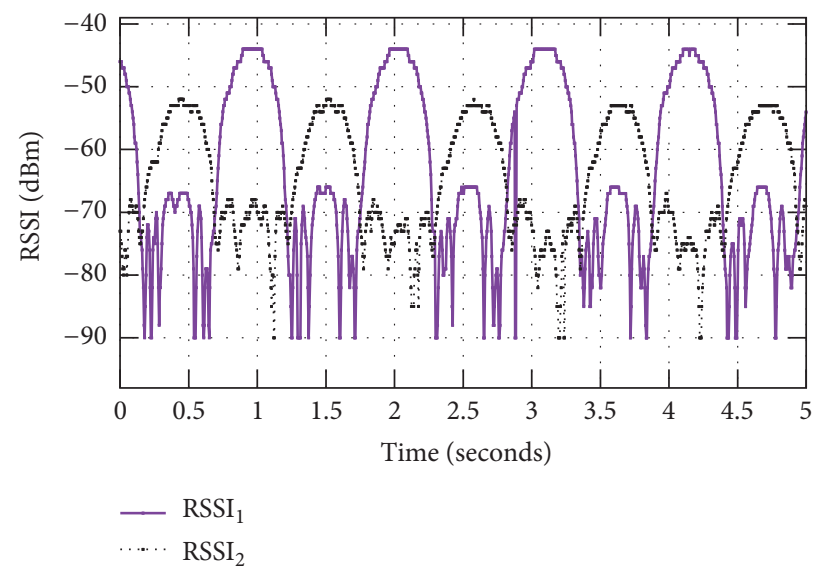

(d) Outdoor + Collar, $270^{\circ}$

FIgURE 8: RSSI versus time in the "Outdoor + Collar" scenario.

In this paper, only the RAP1 and one MAP are adopted to compose the superframe in the beacon-enable mode. Compared to the contention-based methods, TDMA channel access method provides better energy efficiency. Therefore, TDMA method is utilized to upload the data from the sensors and the relay to the hub. As suggested by the IEEE 802.15.6 standard, the TDMA time slots should be assigned to the MAP duration. This is the reason why the MAP is utilized to upload the data from the sensors or the relay to the hub. As for RAP1 period, it precedes MAP to complete the transactions of the connection request and assignment on the basis of CSMA/CA method, which is the suggested approach for RAP in IEEE 802.15.6. These transactions occurring in the RAP1 period are essential for the AA mechanism, as they assign a shared transmission period to a pair of sensors with significant PLD and facilitate the cooperation of relay (the necessity of relay node will be discussed in Section 4.2). Besides, the one-periodic allocation is adopted, where the assigned intervals are reoccurring in every beacon period (superframe). The details of the assignment transactions are illustrated in Figure 11.

The transactions depicted in Figure 11 occur in the RAP1 phase. Initially, the hub broadcasts the beacon frame to the sensors $\left(\mathrm{SN}_{1}\right.$ and $\left.\mathrm{SN}_{2}\right)$ and relay, and all receivers contend for the channel access by a CSMA/CA mechanism. Suppose that $\mathrm{SN}_{1}$ randomly chooses the smallest backoff value. $\mathrm{SN}_{1}$ transmits the connection request packet $\left(\mathrm{Pkt}_{\mathrm{CR}}\right)$ to the hub to request an uplink interval in the MAP. Then, the hub sends a connection allocation packet $\left(\mathrm{Pkt}_{\mathrm{CA}}\right)$ to $\mathrm{SN}_{1}$ to allocate a scheduled uplink interval (SUI). The processes of allocating a SUI to $\mathrm{SN}_{2}$ and the relay are similar. In addition, the relay's scheduled downlink interval (SDI) is allocated to the same period with the sensors' SUI in order to receive the packets from the sensors, which will be detailed in the next subsection. Note that handling the received $\mathrm{Pkt}_{\mathrm{CR}}$ in the hub is the key process for the AA mechanism, which is also marked as three blue rectangles in Figure 11.

The detail of processing $\mathrm{Pkt}_{\mathrm{CR}}$ in the hub is depicted in Algorithm 1. We add a new field item, named PID (Pair Identifier), in the header of $\mathrm{Pkt}_{\mathrm{CR}}$ to assist the relay cooperation. More specifically, the two correlated sensors and their relay have the same PID value, and the SDI of the relay is assigned to overlap with the SUI of the two sensors to receive the packets from the two sensors. NID assigned denotes the NID (Node Identifier) assigned to the node. The hub maintains two independent maps: Map ${ }_{\text {NID }}$ and Map ${ }_{P I D}$. 


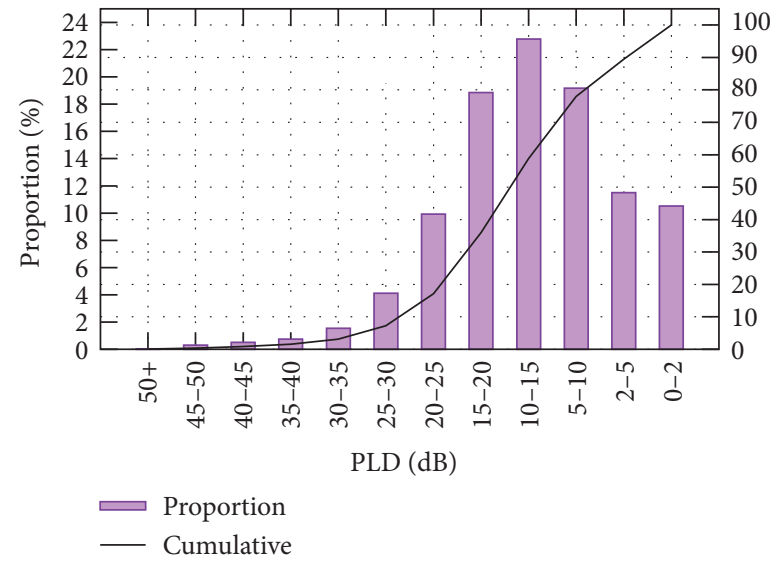

(a) Indoor + Belt

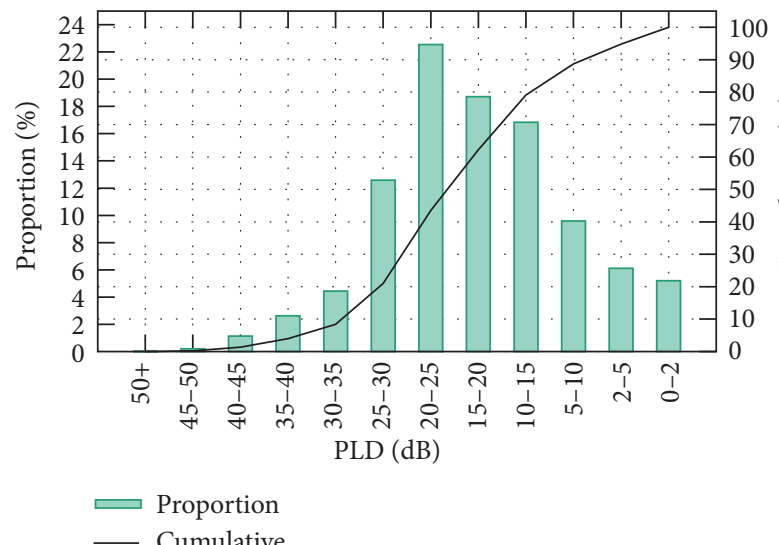

(c) Outdoor + Belt

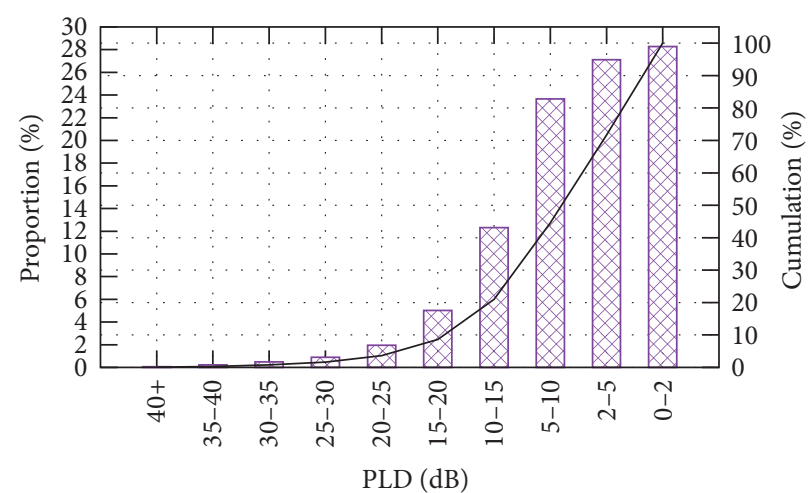

Proportion

- Cumulative

(b) Indoor + Collar

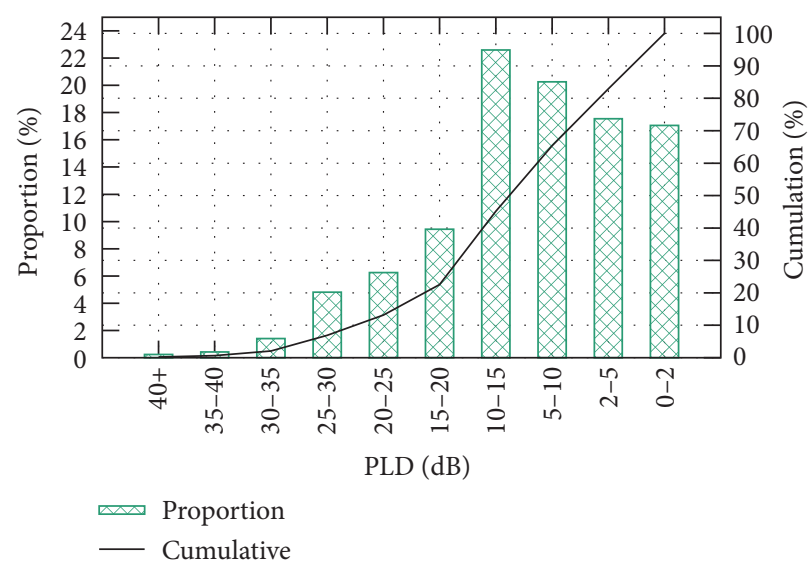

(d) Outdoor + Collar

FIgure 9: The distribution of the PLDs.

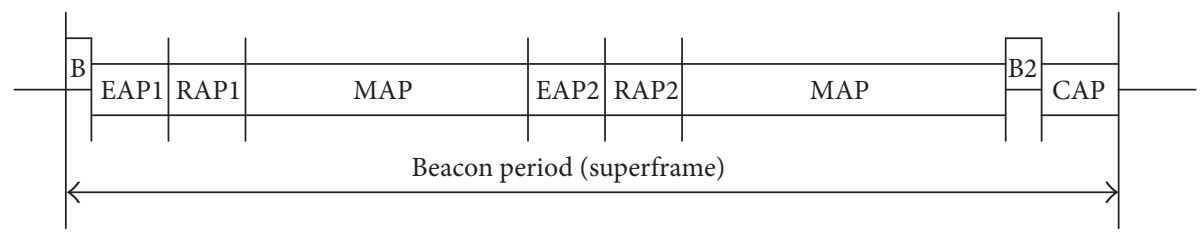

FIGURE 10: Superframe structure of IEEE 802.15.6.

Map $_{\text {NID }}$ contains the mapping between MAC address and NID, and the Map ${ }_{\text {PID }}$ maintains the relation between PID and SUI or SDI. As illustrated in Algorithm 1 (Lines (2)-(5)), a dedicated NID is assigned to each node (sensor or relay) with the help of $\mathrm{Map}_{\mathrm{NID}}$. Consequently, correlated sensors with the same PID share the same SUI by searching Map PID (Lines (7)-(13)). If $\mathrm{Pkt}_{\mathrm{CR}}$ is sent by a relay, the relay node will be allocated with the SDI overlapped with the SUI of the two sensors with the same PID. Meanwhile, an SUI is allocated to the relay to forward data to the hub (as detailed in Lines (15)-(21)). Note that SDI is not essential for sensors since their primary task is uploading the monitoring data to the hub.

After channel allocation, two sensors are allocated to share the same SUI, and the SDI of the relay is overlapped with the SUI of the two sensors. From the perspective of the hub, the two correlated sensors can be looked at as one virtual sensor node. Meanwhile, the beacon period is fixed. Consequently, a longer SUI can be allocated to the sensors, allowing the AA technology to improve the system throughput in walking scenarios. The mathematical analyses will be presented in the next section.

4.2. Cooperative Communication with Analog Network Coding. In this subsection, we first explain the reasons for introducing the relay node. Firstly, by adopting pure AA without employing relay nodes, one of the sensors experiences more severe fading compared to the other one, which is a waste of energy. In other words, AA may achieve the throughput improvement at the cost of additional energy consumption, which is strictly limited in WBANs. Secondly, in the case that 


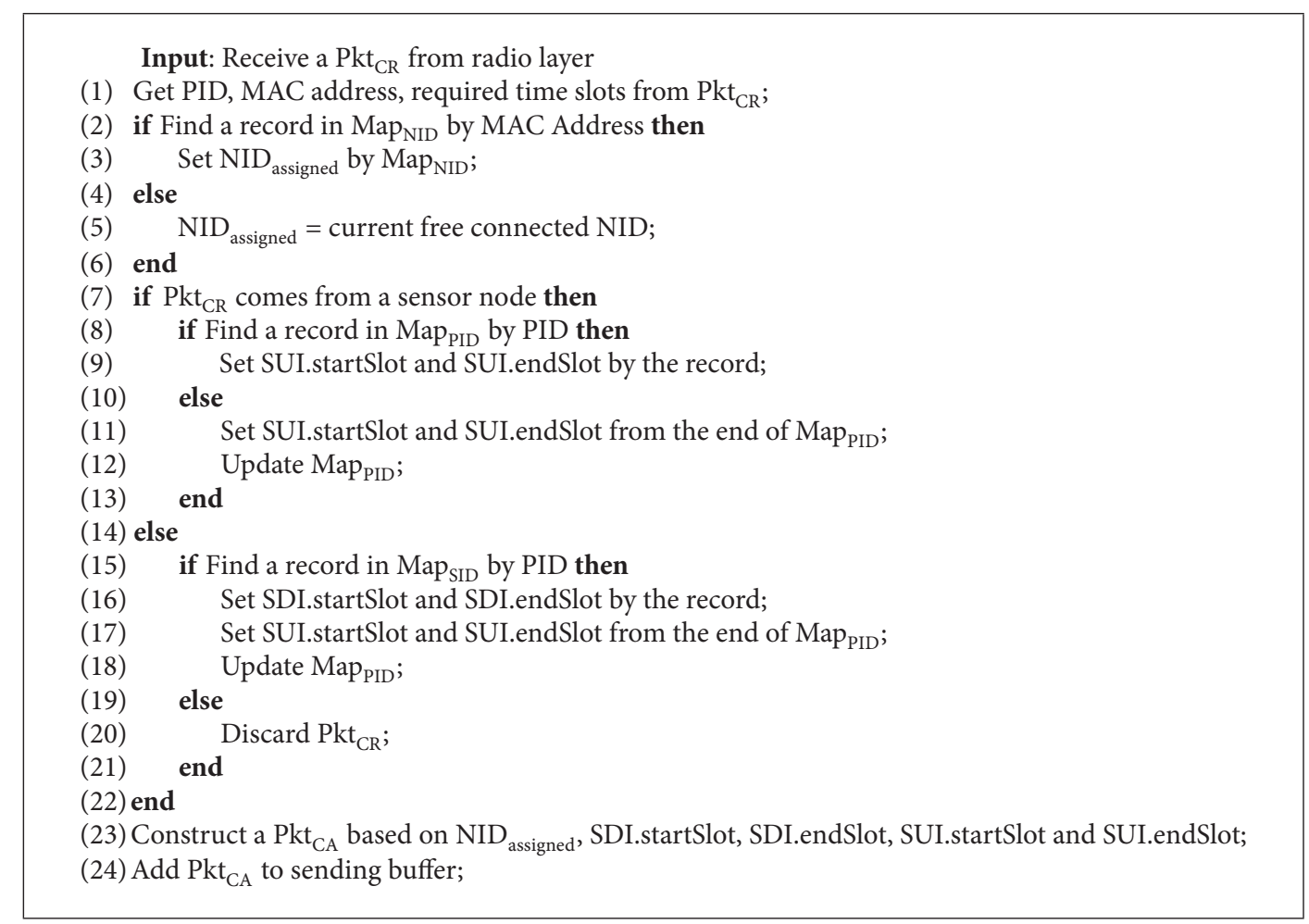

Algorithm 1: Uplink and downlink allocation.

two correlated sensors experience different packets delivery ratios (PDR), AA may cause the sensor with the lower PDR to experience even a worse throughput performance than the conventional TDMA scheme. These two issues can be addressed effectively by deploying a relay node.

Since two sensors broadcast their signals concurrently, the traditional decode-and-forward (DF) relay strategy is impractical. Similarly, the digital network coding (DNC) technology, which requires the relay node to decode and reencode the packets in the MAC layer, is also unrealistic. Given that concurrently transmitted packets from two sensors are mixed naturally at PHY layer and the hub can receive at least one sensor's packet with a high probability, physical-layer network coding (PLNC) technology may be a better choice. In our proposed A3NC, the Analog Network Coding (ANC) technology originally proposed in [35] is adopted. The relay node simply amplifies and forwards the mixed signal to the hub without any decoding, and the hub undertakes the decoding using the mixed signal and one original signal. Perfect synchronization is not required in the ANC technology. Besides, since decoding of packets is not required, the complexity of the relay node is reduced and might be beneficial for the communication security.

Similar to [35], the MSK (Minimum Shift Keying) is adopted as an example modulation scheme to explain the main principle of ANC, for the reason that it has a simple demodulation algorithm as well as good bit error properties. Figure 12 illustrates how to incorporate ANC with cooperative communication. The cooperation procedure can

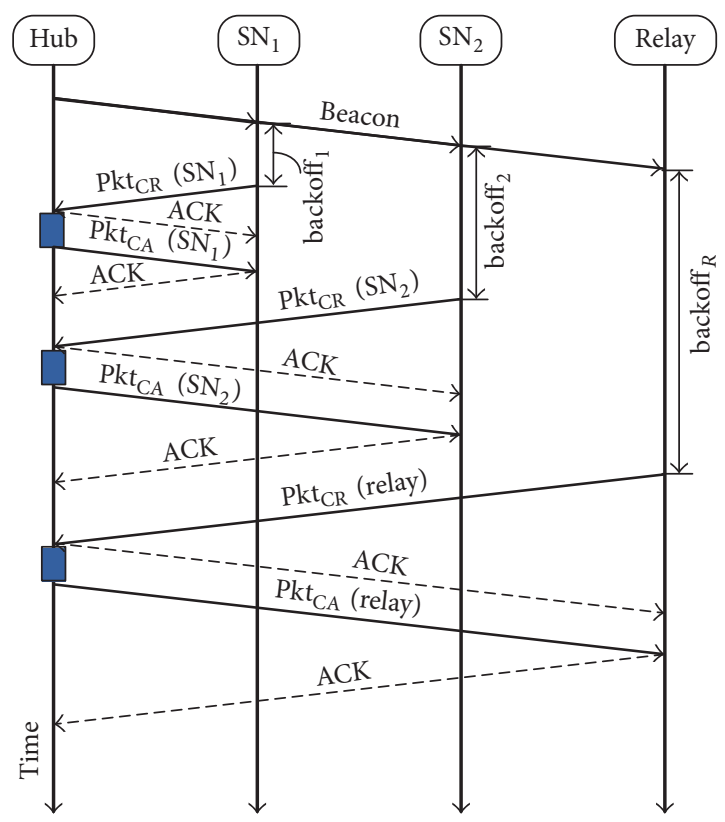

FIGURE 11: Transaction procedure of A3NC in RAP1.

be divided into two phases, which correspond to the SUI of the two sensors (also identical to the SDI of the relay) and the SUI of the relay. As depicted in Figure 12(a), the 


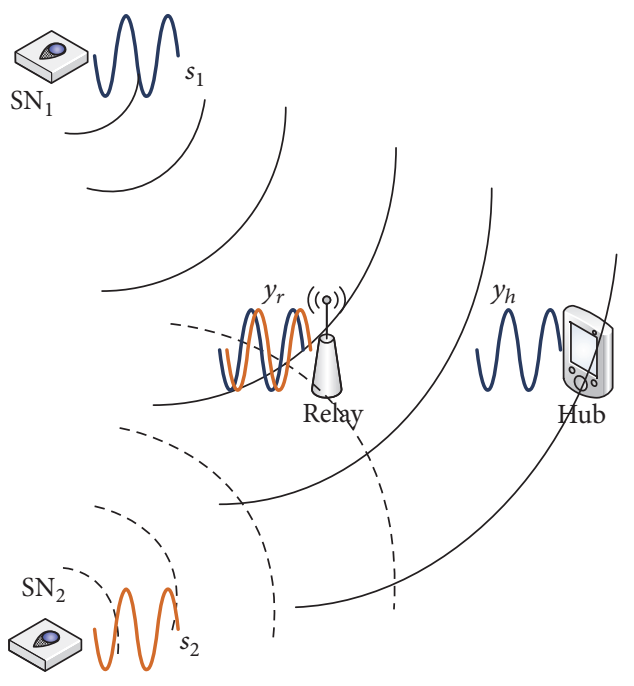

(a)

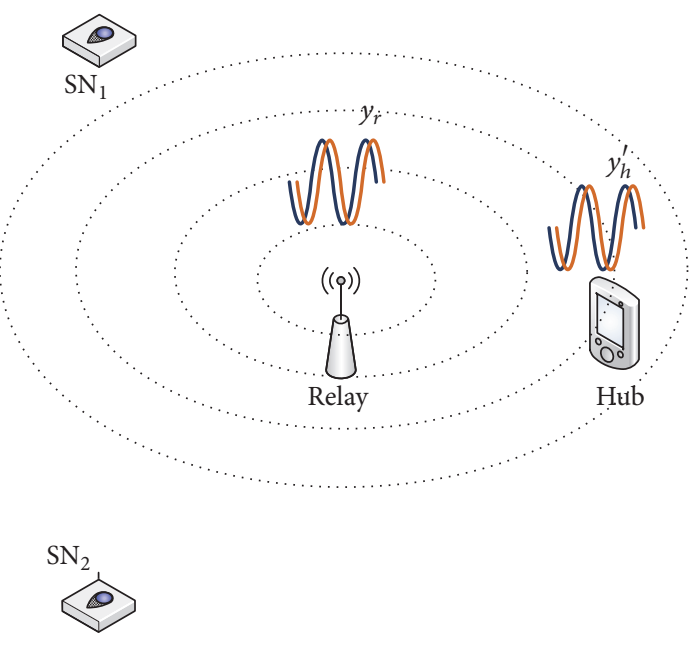

(b)

FIgURE 12: Cooperative communication with ANC. (a) Phase 1: sensors' SUI. (b) Phase 2: relay's SUI.

signals transmitted from $\mathrm{SN}_{1}$ and $\mathrm{SN}_{2}$, respectively, can be represented as

$$
\begin{aligned}
& s_{1}[n]=A_{s}[n] e^{i \theta_{s}[n]}, \\
& s_{2}[n]=B_{s}[n] e^{i \phi_{s}[n]},
\end{aligned}
$$

where $A_{s}[n]$ and $B_{s}[n]$ are the amplitudes of the $n$th sample, and $\theta_{s}[n], \phi_{s}[n]$ are their phases.

If the signal from $\mathrm{SN}_{2}$ (i.e., $s_{2}$ ) experiences a severe fading and the hub successfully receives the signal from $\mathrm{SN}_{1}$, the received signal at the hub, $y_{h}[n]$, can be represented as

$$
y_{h}[n]=h A_{s}[n] e^{i\left(\theta_{s}[n]+\gamma\right)}+w(n),
$$

where $h e^{i \gamma}$ is the complex coefficient for channel $\mathrm{SN}_{1}$-hub and $w(n)$ is the additive Gaussian noise. For brevity, noise term will be ignored in our subsequent formulas.

Meanwhile, the relay node receives and stores the mixed signal $y_{r}[n]$ from two sensors as follows:

$$
y_{r}[n]=h_{1} A_{s}[n] e^{i\left(\theta_{s}[n]+\gamma_{1}\right)}+h_{2} B_{s}[n] e^{i\left(\phi_{s}[n]+\gamma_{2}\right)},
$$

where $h_{1}$ and $\gamma_{1}$ are the channel gain and phase shift, respectively, for the channel $\mathrm{SN}_{1}$-Relay, and $h_{2}$ and $\gamma_{2}$ are those for the channel $\mathrm{SN}_{2}$-Relay.

In the second phase (i.e., the SUI of the relay node), which is shown in Figure 12(b), the relay node amplifies the ANC mixed signal $y_{r}[n]$ and forwards it to the hub. Since the relay node only amplifies and forwards $y_{r}[n]$, for simplicity, we assume the received signal at the hub is the same as the mixed signal at the relay, which can be presented as

$$
y_{h}^{\prime}[n]=A[n] e^{i \theta[n]}+B[n] e^{i \phi[n]},
$$

where $A=h_{1} A_{s}, B=h_{2} B_{s}, \theta[n]=\theta_{s}[n]+\gamma_{1}$, and $\phi[n]=\phi_{s}[n]+\gamma_{2}$. In the complex plane, $y_{h}^{\prime}[n]$ is a sum of two vectors, which have lengths $A$ and $B$, respectively. As shown in Figure 13, the two vectors lie on two circles with radii $A$ and $B$. As proved in [35], there exist two solutions for the pair $(\theta[n], \phi[n])$

$$
\begin{aligned}
& \theta[n]=\arg \left(y_{h}^{\prime}[n]\left(A+B D \pm i B \sqrt{1-D^{2}}\right)\right), \\
& \phi[n]=\arg \left(y_{h}^{\prime}[n]\left(A+A D \mp i A \sqrt{1-D^{2}}\right)\right),
\end{aligned}
$$

where $D=\left(\left|y_{h}^{\prime}[n]\right|^{2}-A^{2}-B^{2}\right) / 2 A B,\left|y_{h}^{\prime}[n]\right|$ is the norm, and arg is the angle of the complex number. Note that, for each solution $\theta[n]$, there is a unique solution for $\phi[n]$. Specifically, if $\theta[n]=\arg \left(y_{h}^{\prime}[n]\left(A+B D+i B \sqrt{1-D^{2}}\right)\right)$, the corresponding solution of $\phi[n]$ is $\arg \left(y_{h}^{\prime}[n]\left(A+A D-i A \sqrt{1-D^{2}}\right)\right)$. In addition, the amplitude of the two signals (i.e., $A$ and $B$ ) can be estimated by the received signal [35].

The next step is to estimate the phase difference between the signals from two sensors, that is, $\theta[n+1]-\theta[n]$ and $\phi[n+$ $1]-\phi[n]$. Corresponding to two potential phase pairs at each sample time, there are four possible pairs of phase differences

$$
\begin{aligned}
& \left(\Delta \theta_{x y}[n], \Delta \phi_{x y}[n]\right) \\
& =\left(\theta_{x}[n+1]-\theta_{y}[n], \phi_{x}[n+1]-\phi_{y}[n]\right) \\
& \quad \forall x, y \in\{1,2\} .
\end{aligned}
$$

Since the channel of $\mathrm{SN}_{1}$-hub is in a "good" condition, the hub knows the signal transmitted from $\mathrm{SN}_{1}, y_{h}[n]$ (cf. (3)). Thus, the hub knows the phase difference $\Delta \theta[n]$. Next, the hub picks $\Delta \theta_{x y}[n]$ that produces the smallest deviation to $\Delta \theta[n]$ as the optimal one, and the matching $\Delta \phi_{x y}[n]$ is the optimal phase difference for the signal from $\mathrm{SN}_{2}$. In the last step, the hub obtains $\mathrm{SN}_{2}$ 's bits based on these estimated phase differences.

Since the focus of this paper is the spatial correlation of on-body channels and the combination of AA and ANC, 


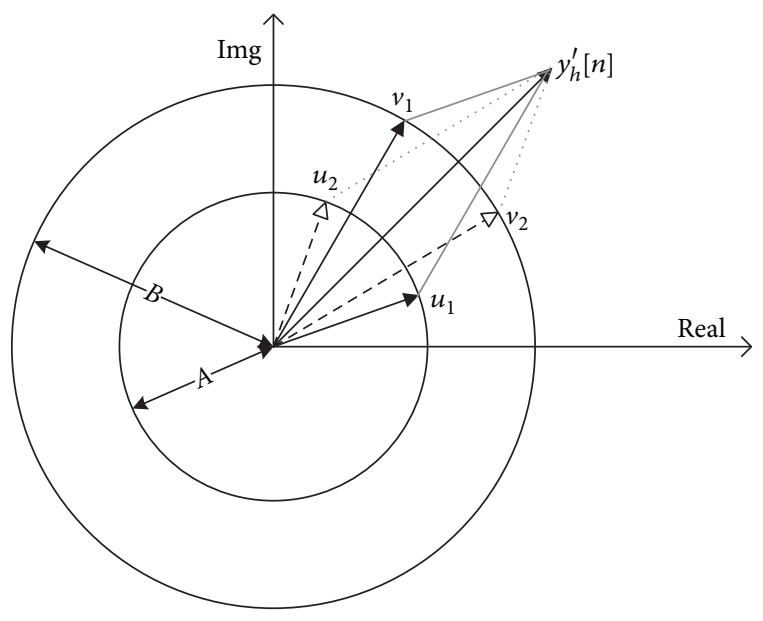

$u_{1}, u_{2}$ : two possible solutions for $\theta[n]$

$v_{1}, v_{2}$ : two possible solutions for $\phi[n]$

FIGURE 13: Two possible solutions for the phase computation.

many other decoding details of ANC, such as the estimation of amplitudes $A$ and $B$ and the alignment of pilot sequences, are omitted in the above example. More details of the ANC technology can be found in [35].

\section{Performance Analysis}

In this section, the performance of the proposed scheme is analyzed and compared to the existing schemes from three perspectives: total throughput, energy efficiency, and throughput balance. The AA mechanism and ANC cooperation are two key components of the proposed A3NC scheme. Three alternative schemes that do not employ both AA and ANC are considered for comparison, and their time slot schedules are depicted in Figure 14.

As shown in Figure 14, suppose a certain period $T_{c}$ is allocated to the two sensors and the corresponding relay node. In the conventional TDMA scheme, denoted as ConTDMA (Figure 14(a)), $T_{c}$ is split into two for the SUI of the two sensors, whereas when AA mechanism is employed without the cooperative communication part of $\mathrm{A} 3 \mathrm{NC}$, denoted as Only-AA (Figure 14(b)), both sensors share the whole $T_{c}$ concurrently. Next, there are two different approaches to assign the SUI of the relay. First, when the relay node forwards the digital network coding (DNC) packets to the hub in the third time interval, each of the two sensors possesses an exclusive SUI. This approach is named as TDMA-DNC, in which $T_{c}$ is divided into three identical parts. The second one is our proposed scheme A3NC (Figure 14(d)); the two sensors are assigned to a shared SUI, and the ANC is adopted as the relay technology to improve the performance.

5.1. Throughput Analysis. Before analyzing the upload throughput of the four schemes, it is necessary to examine $e$ in more detail. As introduced in Section 2, $e$ denotes the packet error ratio (PER) caused by signal collision, which only occurs when two sensors perform simultaneous transmissions in the MAP duration, so $e$ only exist in Only$\mathrm{AA}$ and $\mathrm{A} 3 \mathrm{NC}$. As will be presented in the next section, $e$ in the typical walking scenario would be small enough (about 0.022 ) to be ignored. However, in order to explore the performance of $\mathrm{A} 3 \mathrm{NC}$ in other situations, rather than being limited to walking scenarios, we consider $e$ as a variable parameter in both mathematical and simulation analyses. Besides, we assume $e$ for the two sensors to be identical due to symmetry.

5.1.1. Conventional TDMA. We first consider the Con-TDMA scheme without the cooperation of the relay node. In this scheme, every sensor is scheduled with a dedicated uplink interval. The expectation of upload throughput of $\mathrm{SN}_{1}$, denoted as $E\left(U_{1}\right)$ (Kbps), can be calculated as

$$
\begin{aligned}
E\left(U_{1}\right)_{\text {Con-TDMA }} & =\left(R \times \frac{1}{2} T_{c} \times \frac{1}{L_{p}} \times \beta_{1} \times L_{p}\right) \times \frac{1}{T_{s}} \\
& =\frac{1}{2} \frac{R T_{c} \beta_{1}}{T_{s}} .
\end{aligned}
$$

Since only two sensors share the time interval, every sensor occupies $(1 / 2) T_{c}$ upload interval; $R \times(1 / 2) T_{c} \times 1 / L_{p}$ is the number of packets transmitted in one beacon period by $\mathrm{SN}_{1}$. $\left(R \times(1 / 2) T_{c} \times 1 / L_{p} \times \beta_{1} \times L_{p}\right)$ is the amount of bits received by the hub. The calculation for the upload throughput of $\mathrm{SN}_{2}$, that is, $E\left(U_{2}\right)$, is similar. Thus, the expectation of the sum of upload throughputs of the two sensors can be expressed as

$$
E\left(U_{1}+U_{2}\right)_{\text {Con-TDMA }}=\frac{1}{2} \frac{R T_{c}}{T_{s}}\left(\beta_{1}+\beta_{2}\right) .
$$

5.1.2. Only-AA. In the Only-AA scheme, since two sensors transmit simultaneously, the packet errors caused by signal collisions should be considered. The actual PDRs for $\mathrm{SN}_{1}$ and $\mathrm{SN}_{2}$ are $\left(\beta_{1}-e\right)$ and $\left(\beta_{2}-e\right)$, respectively. Accordingly, the throughput of the two sensors can be expressed as

$$
\begin{aligned}
E\left(U_{1}+U_{2}\right)_{\text {Only-AA }} & =\frac{R T_{c}\left(\beta_{1}-e\right)}{T_{s}}+\frac{R T_{c}\left(\beta_{1}-e\right)}{T_{s}} \\
& =\frac{R T_{c}}{T_{s}}\left(\beta_{1}+\beta_{2}-2 e\right) .
\end{aligned}
$$

5.1.3. TDMA + DNC. The TDMA + DNC scheme is a classical approach that adopts DNC as the cooperative technology. As Figure 14(c) shows, the relay node occupies the third time interval to transmit the network coding packets to the hub. Since the packet loss in the first two time slots may be recovered in the third time slot, the probability of effective cooperative communication should be taken into account to calculate the actual PDR (denoted as $\mathscr{B}_{1}$ and $\mathscr{B}_{2}$ ). If the result of whether the hub receives the packets from $\mathrm{SN}_{1}$ is represented by a discrete random variable (DRV) $X$, then let $X=S$ denote the situation that the hub receives the packets from $\mathrm{SN}_{1}$ successfully, and $X=F$ represents the failure of the hub to receive the packets from $\mathrm{SN}_{1}$. Similarly, DRV $Y$ and $R$ 


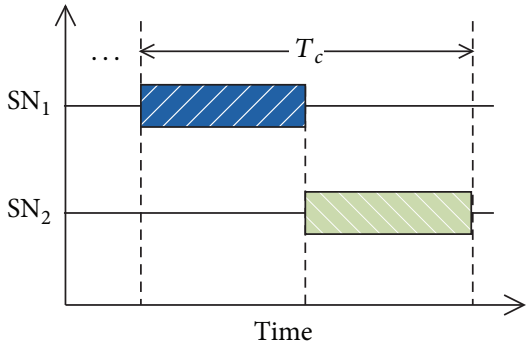

(a) Con-TDMA

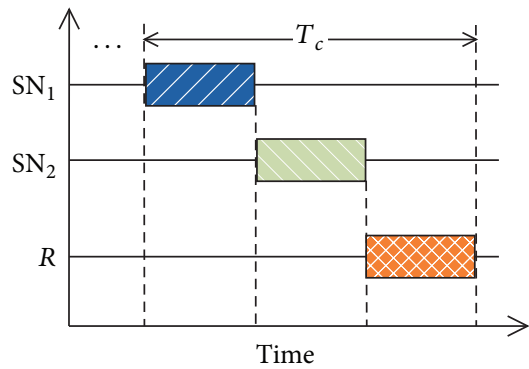

(c) TDMA + DNC

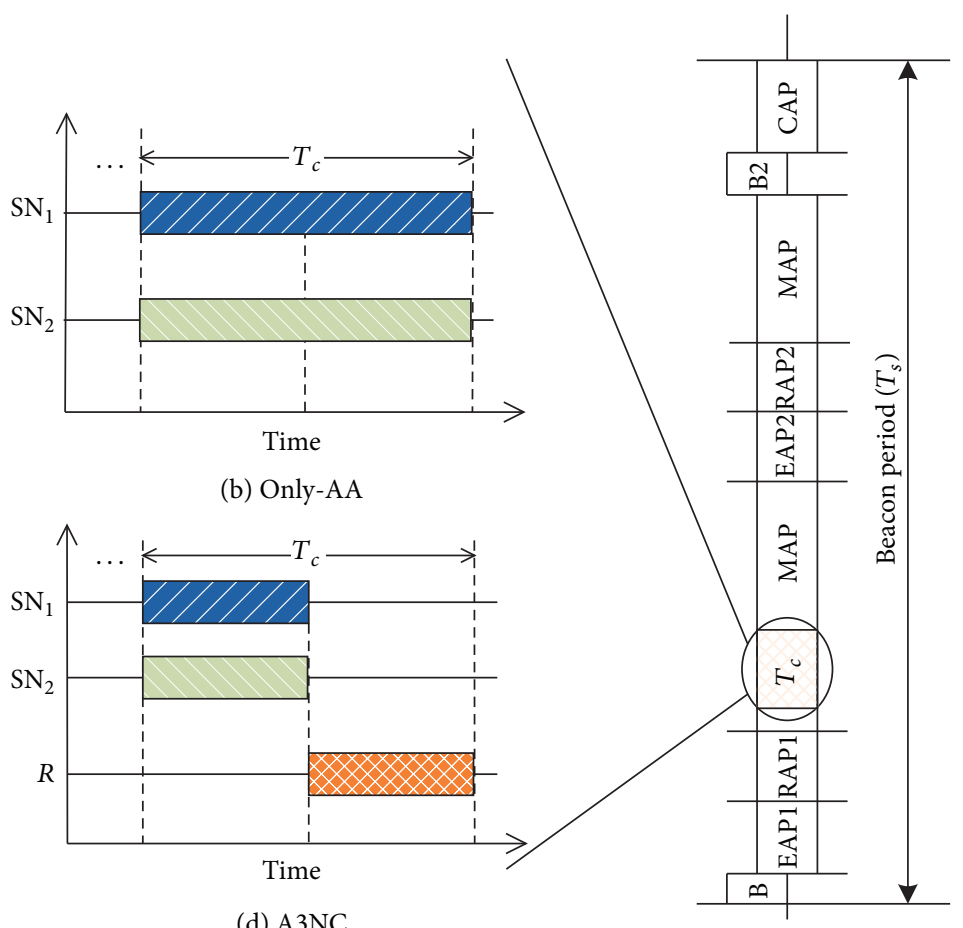

(d) $\mathrm{A} 3 \mathrm{NC}$
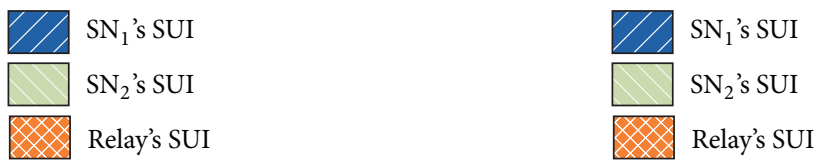

FIGURE 14: Slot schedules of four schemes.

denote the situations for $\mathrm{SN}_{2}$ and the relay node, respectively. Hence, $\mathscr{B}_{1}$ and $\mathscr{B}_{2}$ can be calculated as

$$
\begin{aligned}
& \mathscr{B}_{1}=P_{X, Y}(S, F)+P_{X, Y}(S, S)+P_{X, Y}(F, S) P_{R}(S), \\
& \mathscr{B}_{2}=P_{X, Y}(F, S)+P_{X, Y}(S, S)+P_{X, Y}(S, F) P_{R}(S),
\end{aligned}
$$

where $P_{X, Y}$ is the joint probability that considers both DRV $X$ and $Y$, and $P_{R}$ denotes the probability of whether the hub receives the packets from the relay node. It is worth noting that $\beta_{1}=P_{X, Y}(S, F)+P_{X, Y}(S, S), \beta_{2}=P_{X, Y}(F, S)+P_{X, Y}(S, S)$, and $\beta_{r}=P_{R}(S)$. Accordingly, $\mathscr{B}_{1}$ and $\mathscr{B}_{2}$ can be expressed as

$$
\begin{aligned}
& \mathscr{B}_{1}=\beta_{1}+\left(\beta_{2}-P_{X, Y}(S, S)\right) \beta_{r}, \\
& \mathscr{B}_{2}=\beta_{2}+\left(\beta_{1}-P_{X, Y}(S, S)\right) \beta_{r} .
\end{aligned}
$$

For a general wireless network, it is not straightforward to calculate the probability that the packets from two sensors are received by the hub, that is, $P_{X, Y}(S, S)$. However, since we mainly consider the walking scenarios, $P_{X, Y}(S, S)$ can be approximated as

$$
P_{X, Y}(S, S) \approx \begin{cases}0 ; & \beta_{1}+\beta_{2} \leq 1 \\ \left(\beta_{1}+\beta_{2}\right)-1 ; & \beta_{1}+\beta_{2}>1\end{cases}
$$

We derive the above approximation based on the following observations. Compared to the walking cycle (about $1000 \mathrm{~ms}$, see Section 3.2), the superframe (about $100 \mathrm{~ms}$ ) is relatively short. Moreover, the two upload intervals assigned to the two sensors are typically adjacent. So, based on the statistical results in Section 3, it is reasonable to assume that the PLD between the two channels remains significant and stable in one superframe. To make the approximation in (13) clear, suppose that each sensor only sends one packet in one superframe. Accordingly, in the case of $\beta_{1}+\beta_{2} \leq 1$, the hub tends to receive only one packet from either $\mathrm{SN}_{1}$ or $\mathrm{SN}_{2}$ with a high probability in one superframe, instead of receiving packets from both sensors simultaneously. On the other hand, in the case of $\beta_{1}+\beta_{2}>1$, the received signal strength from the "bad" channel is still strong enough to decode, regardless of the big PLDs between the two channels. Note that the latter case is normally caused by the increase of Tx power of sensors. Therefore, based on ((12)-(13)), the expected overall throughput can be obtained as

$$
\begin{aligned}
& E\left(U_{1}+U_{2}\right)_{\mathrm{TDMA}+\mathrm{DNC}}=\frac{1}{3} \frac{R T_{c} \mathscr{B}_{1}}{T_{s}}+\frac{1}{3} \frac{R T_{c} \mathscr{B}_{2}}{T_{s}} \\
& \approx \begin{cases}\frac{1}{3} \frac{R T_{c}}{T_{s}}\left(\beta_{1}+\beta_{2}\right)\left(1+\beta_{r}\right) ; & \beta_{1}+\beta_{2} \leq 1, \\
\frac{R T_{c}}{3} \frac{R}{T_{s}}\left(\left(\beta_{1}+\beta_{2}\right)\left(1-\beta_{r}\right)+2 \beta_{r}\right) ; & \beta_{1}+\beta_{2}>1 .\end{cases}
\end{aligned}
$$


5.1.4. A3NC. As for the A3NC scheme, two sensors broadcast the data packets simultaneously in the first half period, and the ANC packet is relayed in the second half. This situation can be considered as an integration of Only-AA and TDMA + DNC schemes. When calculating the actual PDRs, denoted as $\widetilde{\mathscr{B}}_{1}$ and $\widetilde{\mathscr{B}}_{2}$, both the cooperative communication and the packet loss caused by signal collision should be taken into account. The actual PDRs for $\mathrm{SN}_{1}$ and $\mathrm{SN}_{2}$ are expressed as

$$
\begin{aligned}
& \widetilde{\mathscr{B}}_{1}=\left(\beta_{1}-e\right)+\left(\beta_{2}-e\right) \beta_{r}, \\
& \widetilde{\mathscr{B}}_{2}=\left(\beta_{2}-e\right)+\left(\beta_{1}-e\right) \beta_{r} .
\end{aligned}
$$

The first parts of (15) refer to the PDRs for the first half timeslot, and the second parts are the PDRs with the help of the relay node. Consequently, the upload throughput of the whole network can be written as

$$
\begin{aligned}
E\left(U_{1}+U_{2}\right)_{\mathrm{A} 3 \mathrm{NC}} & =\frac{1}{2} \frac{R T_{c} \widetilde{\mathscr{B}}_{1}}{T_{s}}+\frac{1}{2} \frac{R T_{c} \widetilde{\mathscr{B}}_{2}}{T_{s}} \\
& =\frac{1}{2} \frac{R T_{c}}{T_{s}}\left(\beta_{1}+\beta_{2}-2 e\right)\left(1+\beta_{r}\right) .
\end{aligned}
$$

As a summary, we list some important observations by comparing the analytical results of the four schemes.

Observation 1. If the packets error ratio caused by signal collision is below the quarter of $\beta_{1}+\beta_{2}$, the throughput performance of Only-AA is better than that of Con-TDMA scheme. That is

$$
E\left(U_{1}+U_{2}\right)_{\text {Only-AA }} \begin{cases}>E\left(U_{1}+U_{2}\right)_{\text {Con-TDMA }} ; & e<\frac{1}{4}\left(\beta_{1}+\beta_{2}\right), \\ \leq E\left(U_{1}+U_{2}\right)_{\text {Con-TDMA }} ; & \text { o.w. }\end{cases}
$$

Observation 2. The upload throughput performance of A3NC is always under that of Only-AA, because the PDR of the relay channel cannot exceed one; that is, $\beta_{r} \leq 1$.

$$
E\left(U_{1}+U_{2}\right)_{\mathrm{A} 3 \mathrm{NC}} \leq E\left(U_{1}+U_{2}\right)_{\text {Only-AA }} .
$$

Observation 3. The comparison between Con-TDMA and TDMA + DNC is complicated. If $\beta_{1}+\beta_{2} \leq 1$, we have

$$
E\left(U_{1}+U_{2}\right)_{\mathrm{TDMA}+\mathrm{DNC}} \begin{cases}>E\left(U_{1}+U_{2}\right)_{\mathrm{Con}-\mathrm{TDMA}} ; & \beta_{r}>\frac{1}{2}, \\ \leq E\left(U_{1}+U_{2}\right)_{\mathrm{Con}-\mathrm{TDMA}} ; & \beta_{r} \leq \frac{1}{2}\end{cases}
$$

If $1 \leq \beta_{1}+\beta_{2} \leq 4 / 3$ and we denote $Q=\left(\beta_{1}+\beta_{2}\right) /(4-$ $\left.2\left(\beta_{1}+\beta_{2}\right)\right)$, the relationship between these two schemes can be expressed as

$$
E\left(U_{1}+U_{2}\right)_{\mathrm{TDMA}+\mathrm{DNC}} \begin{cases}>E\left(U_{1}+U_{2}\right)_{\mathrm{Con}-\mathrm{TDMA}} ; & \beta_{r}>\mathrm{Q}, \\ \leq E\left(U_{1}+U_{2}\right)_{\mathrm{Con}-\mathrm{TDMA}} ; & \beta_{r} \leq \mathrm{Q} .\end{cases}
$$

If $\beta_{1}+\beta_{2}>4 / 3$, the throughput performance of TDMA + DNC cannot exceed that of Con-TDMA. That is,

$$
E\left(U_{1}+U_{2}\right)_{\mathrm{TDMA}+\mathrm{DNC}}<E\left(U_{1}+U_{2}\right)_{\mathrm{Con}-\mathrm{TDMA}} .
$$

Observation 4. When $e<(1 / 6)\left(\beta_{1}+\beta_{2}\right)$, A3NC achieves a better throughput performance than TDMA + DNC:

$$
E\left(U_{1}+U_{2}\right)_{\mathrm{A} 3 \mathrm{NC}}>E\left(U_{1}+U_{2}\right)_{\mathrm{TDMA}+\mathrm{DNC}} .
$$

It can be seen that both $\mathrm{A} 3 \mathrm{NC}$ and Only-AA outperform the conventional schemes when the values of specific parameters (i.e., $e, \beta_{r}$, and $\beta_{1}+\beta_{2}$ ) satisfy certain conditions. Detailed performance evaluation based on walking datasets will be provided in the next section.
In addition, if the packets losses due to signal collisions and failed cooperation are small, that is, $e \approx 0$ and $\beta_{r} \approx 1$, we can derive the upper bounds for the four schemes in the walking scenarios. For brevity, denote $M=\left(R T_{c} / T_{s}\right)\left(\beta_{1}+\right.$ $\beta_{2}$ ). The upper bounds of upload throughput are depicted in Table 2. We notice that the upper bounds for Only-AA and $\mathrm{A} 3 \mathrm{NC}$ are the same, that is, $\left(R T_{c} / T_{s} L_{p}\right)\left(\beta_{1}+\beta_{2}\right)$, which achieves 2 and 1.5 throughput gain in comparison with ConTDMA and TDMA + DNC, respectively.

5.2. Energy Efficiency Analysis. An effective MAC layer method for energy saving is prolonging sleep time of the sensors, which also means completing data transmission in a shorter active time. Generally, recharging and changing the battery in a hub are easier than in the sensors. The relay node is deployed out of human body. Hence, in this paper, the energy efficiencies of hub and relay are not considered. Moreover, due to the behaviors of hub, relay and sensor nodes are repeated in each beacon period, and we take the active time in one beacon period as an example. Note that the active state of the radio includes both $\mathrm{Tx}$ (transmission) and $\mathrm{Rx}$ (reception) states. Assuming the working power of sensors remains the same for both $\mathrm{Rx}$ and $\mathrm{Tx}$ states, the energy efficiency (EE) in Kbits/Joule can be defined as the ratio of the amount of uploading data and the power consumed in one beacon period:

$$
\mathrm{EE}=\frac{E\left(U_{1}+U_{2}\right) T_{s}}{T_{\text {active }} \mathscr{P}_{w}},
$$

where $T_{\text {active }}$ is the active time of the two sensors in one beacon period, and $\mathscr{P}_{w}$ is the working power of the sensor. $E\left(U_{1}+U_{2}\right)$ is the expected sum of the upload throughput of the two sensors, and it has been analyzed before. We now focus on the $T_{\text {active }}$, which can be calculated from Figure 14 . 
TABLE 2: The upper bound of throughput.

\begin{tabular}{lcccc}
\hline & Con-TDMA & Only-AA & TDMA + DNC & A3NC \\
\hline $\begin{array}{l}\text { Expectation of } \\
\text { throughput }\end{array}$ & $\frac{1}{2} M$ & $M$ & $\frac{2}{3} M$ & $M$ \\
\hline
\end{tabular}

TABLE 3: Energy efficiency comparison.

\begin{tabular}{lcccc}
\hline & Con-TDMA & Only-AA & TDMA + DNC & A3NC \\
\hline$T_{\text {active }}$ & $T_{c}$ & $2 T_{c}$ & $\frac{2}{3} T_{c}$ & $T_{c}$ \\
EE & $\frac{1}{2} N$ & $\frac{1}{2} N$ & $N$ & $N$ \\
\hline
\end{tabular}

TABLE 4: Balance factor comparison.

\begin{tabular}{|c|c|c|c|c|}
\hline & Con-TDMA & Only-AA & TDMA + DNC & $\mathrm{A} 3 \mathrm{NC}$ \\
\hline $\mathrm{BF}$ & $\left|\log _{2} \frac{\beta_{1}}{\beta_{2}}\right|$ & $\log _{2} \frac{\beta_{1}-e}{\beta_{2}-e}$ & 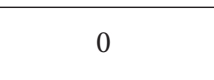 & 0 \\
\hline
\end{tabular}

Similar to the analysis of throughput, if $e=0, \beta_{r}=1$, and we denote $N=R\left(\beta_{1}+\beta_{2}\right) / \mathscr{P}_{w}$, the upper bounds of EE are summarized in Table 3 . From Table 3 , the two cooperative schemes (TDMA + DNC and A3NC) achieve a twofold EE gain, compared to Con-TDMA and Only-AA.

Combining the results in Tables 2 and 3, we find that the A3NC scheme not only achieves the highest throughput upper bound but also retains the best energy efficiency. In other words, the A3NC scheme provides a better tradeoff between network throughput and energy efficiency.

5.3. Throughput Balance Analysis. The above analyses consider the total upload throughput of the two sensors. In this subsection, the throughput disparity between the two sensors (i.e., $E\left(U_{1}\right)$ and $\left.E\left(U_{2}\right)\right)$ is taken into account, instead of only considering their sum. The aim is to assess whether the proposed A3NC scheme may result in a severe throughput imbalance between the two sensors, for example, one sensor being completely starved. We define a new parameter called the balance factor $(\mathrm{BF})$ to evaluate whether the transmission scheme can achieve a reasonable throughput balance between the two sensors. The balance factor, BF, is defined by the following equation:

$$
\mathrm{BF}=\left|\log _{2} \frac{E\left(U_{1}\right)}{E\left(U_{2}\right)}\right|,
$$

where $E\left(U_{1}\right)$ and $E\left(U_{2}\right)$ are the expected throughput of $\mathrm{SN}_{1}$ and $\mathrm{SN}_{2}$, respectively. If $\mathrm{BF}=0$, the two sensors achieve the same throughput, no matter whether $\beta_{1}$ is equal to $\beta_{2}$ or not. On the other hand, the larger the $\mathrm{BF}$ is, the bigger the throughput disparity between the two sensors is. Table 4 summarizes the $\mathrm{BF}$ when the relay node achieves a perfect cooperation, that is, $\beta_{r}=1$.

As shown in Table 4, the BFs of the two cooperative schemes (TDMA + DNC and A3NC) are zero, but the BFs of other two noncooperative schemes vary with the ratio of two actual PDRs. That is because, with the help of the relay, the hub can decode the packet sent through the "bad" channel by receiving a cooperative packet from the "good" channel, which balances the throughput of the two sensors. Therefore, TDMA + DNC and A3NC achieve a better throughput balance, compared to the schemes without relay nodes.

In summary, the proposed $\mathrm{A} 3 \mathrm{NC}$ scheme achieves a better throughput in comparison with the schemes without AA method. Meanwhile, due to the contribution of relay node, the A3NC also achieves a better energy efficiency and throughput balance compared to the schemes without the relay.

\section{Performance Evaluation}

In this section, simulation results will be provided to show the performance of the A3NC scheme in terms of total throughput, energy efficiency, and throughput balance.

6.1. Simulation Model and Configurations. The protocol stacks from PHY layer to application layer are developed based on the Castalia framework [36] in OMNeT++. All the important default parameters of protocol stacks and hardware are listed in Table 5 . The parameters of the routing layer are not listed in the table because the routing layer in the model only forwards packets between the application layer and MAC layer. Besides, the initial energy of the sensor is set to 2430 Joules, which is the average energy of one CR2032 lithium button battery with 3.0 voltages and $225 \mathrm{mAh}$ capacity. For the fairness of comparison, the SUI of the sensors and the SUI of the relay are set up to different values in different schemes to make sure that the overall time $\left(T_{c}\right)$ remains $120 \mathrm{~ms}$ (as depicted in Figure 14).

In this paper, both the path loss datasets and a simulated network model are utilized to construct channel-related parameters. First, the datasets collected from experiments are imported to simulate the variation of the path loss in time. However, these datasets only cover a limited number of walking scenarios, and the critical parameters, that is, $\beta_{i}, \beta_{r}$, and $e$, in each scenario are fixed. In order to verify the theoretical analyses results mentioned in Section 5, a simulated network model should also be used to adjust $\beta_{i}, \beta_{r}$, and $e$. The combination of the experimental datasets and simulated network model constructed a more flexible simulation platform.

Figure 15 presents the simulated network model. SP1 and SP2 are the starting points for the two sensors. The reciprocating motion of the two sensors is simulated by importing the trace file, which records the path loss sequence during the walking activities. We now explain how to tune $\beta_{i}, \beta_{r}$, and $e$. The "Outdoor + Belt" scenario with the transceivers bound in 0 degree is picked as the typical one, and the corresponding path loss dataset is imported into the network model. First of all, the average PDR for the channel $\mathrm{SN}_{i}$-hub (i.e., $\beta_{i}$ ) can be changed by modifying the Tx power. As shown in Figure 16, when the Tx power of the two sensors decreases, the sum of $\beta_{1}$ and $\beta_{2}$ also diminishes. Moreover, because the two sensors have the same Tx power, $\beta_{1}$ and $\beta_{2}$ are also approximately identical.

The average PDR of channel relay-hub (i.e., $\beta_{r}$ ) can be tuned by setting up a parameter (named PDR_Relay) in $\mathrm{OMNeT}++$ to modify the behavior of the relay node. At 
TABLE 5: Simulation parameters.

\begin{tabular}{|c|c|}
\hline Parameter & Value \\
\hline \multicolumn{2}{|c|}{ Radio layer } \\
\hline dataRate & $512 \mathrm{kbps}$ \\
\hline Bandwidth & $20 \mathrm{MHz}$ \\
\hline noiseFloor & $-101 \mathrm{dBm}$ \\
\hline CCAthreshold & $-95 \mathrm{dBm}$ \\
\hline Tx Power & $-38 \mathrm{dBm}$ \\
\hline Rx state energy consumption & $3.1 \mathrm{~mW}$ \\
\hline modulationType & DIFFBPSK \\
\hline carrierFreq & $2400.0 \mathrm{MHz}$ \\
\hline Sensitivity & $-91 \mathrm{dBm}$ \\
\hline symbolsForRSSI & 8 bits \\
\hline Tx state energy consumption & $2.9 \mathrm{~mW}$ \\
\hline Idle state energy consumption & $0.05 \mathrm{~mW}$ \\
\hline \multicolumn{2}{|c|}{ MAC layer } \\
\hline beaconPeriodLength & $160 \mathrm{~ms}$ \\
\hline SlotLength & $5 \mathrm{~ms}$ \\
\hline Data's ACKType & N_ACK \\
\hline RAP1Length & $40 \mathrm{~ms}$ \\
\hline pTIFS & $0.03 \mathrm{~ms}$ \\
\hline Control's ACK & I_ACK \\
\hline \multicolumn{2}{|c|}{ Others } \\
\hline Sensor's initialEnergy & $2430 \mathrm{~J}$ \\
\hline simulationTime & $50 \mathrm{~s}$ \\
\hline
\end{tabular}

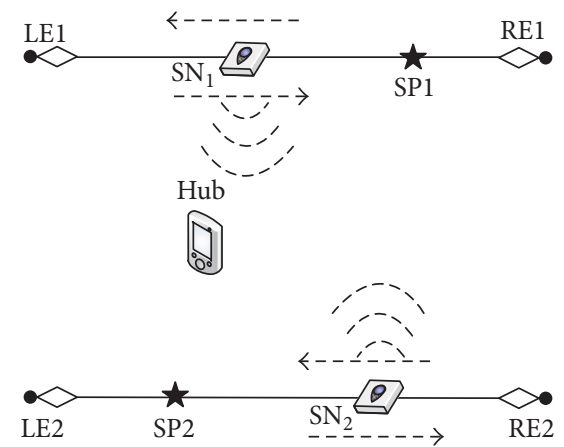

FIGURE 15: Simulated network model in OMNeT++.

last, the average PER due to collisions (i.e., $e$ ) is controlled by changing the relative position between the two sensors' start points (SP1 and SP2). From Figure 15, intuitively, the interference between the two sensors is maximized (i.e., maximum $e$ ), if both SP1 and SP2 are in the left endpoint of their motion trajectories (i.e., LE1 and LE2). But, if SP1 is on LE1 and SP2 is on RE2, which is a common situation in walking scenarios, $e$ reaches its minimum value. Accordingly, we can change the value of $e$ by changing the two start points of the sensors. Specifically, $e$ can be changed by scrolling the path loss sequence of $\mathrm{SN}_{2}$ while remaining the path loss sequence of $\mathrm{SN}_{1}$. As the sampling rate is fixed, the scrolling of path loss sequence can also be denoted as the time shifting compared to the normal arm swing in the walking scenarios.

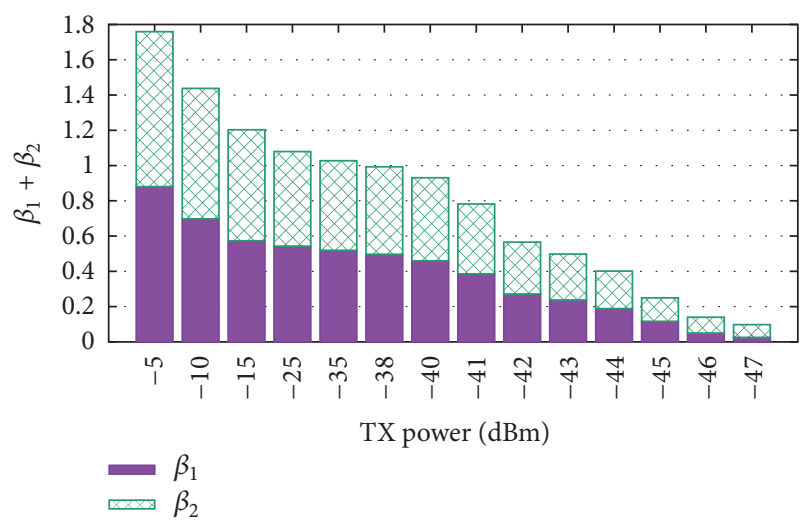

FIgURE 16: $\beta_{1}+\beta_{2}$ versus TX power.

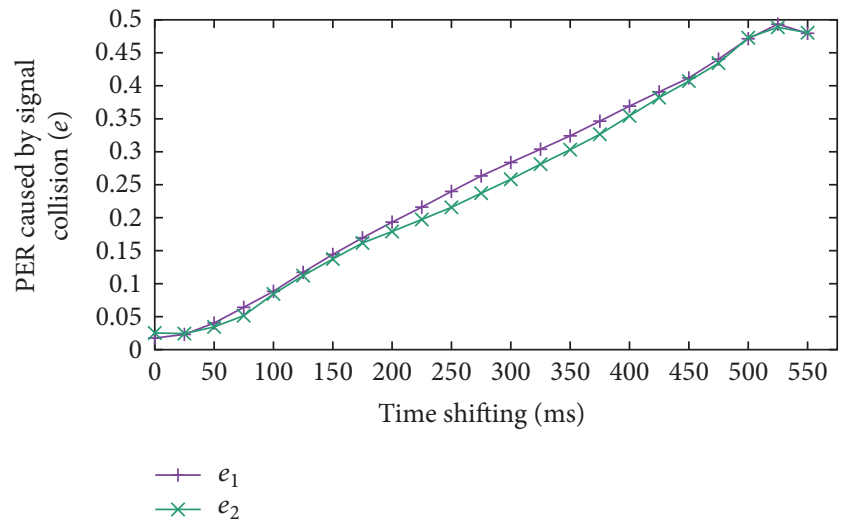

FIGURE 17: $e$ versus time shifting.

Figure 17 presents the curve of $e$ versus time shifting, when the Tx power of the two sensors is set to $-38 \mathrm{dBm}$ and $\beta_{1}+\beta_{2}$ is approximately one. The case of "time shifting $=0$ " corresponds to the normal swing motion of arm in walking scenarios. As shown in Figure 17, the average PER due to collisions for normal walking scenarios is about 0.022 . On the contrary, when the time shifting reaches $525 \mathrm{~ms}$, both $e_{1}$ and $e_{2}$ reach the maximum (about 0.5 ). In addition, it also implies that the cycle for chosen walking scenarios is around $1050 \mathrm{~ms}$. It is worth noting that the change of Tx power may also lead to the variation of $e$.

\subsection{Simulation Results}

6.2.1. Throughput. Figure 18 shows the average total throughput of the proposed $\mathrm{A} 3 \mathrm{NC}$ scheme in comparison with the Con-TDMA, Only-AA, and TDMA + DNC schemes in four typical walking scenarios, with consideration of four different deployment directions on the wrists. The Tx power and $\beta_{r}$ are set at $-38 \mathrm{dBm}$ and 0.9 , respectively. The "total throughput" here means the sum of the average upload throughput of the two sensors.

As shown in Figure 18, the performances of Only-AA and A3NC are slightly below that of Con-TDMA and TDMA + DNC in the "Indoor + Collar" scenario. The reason is that, compared to the cases where the hub is deployed in 


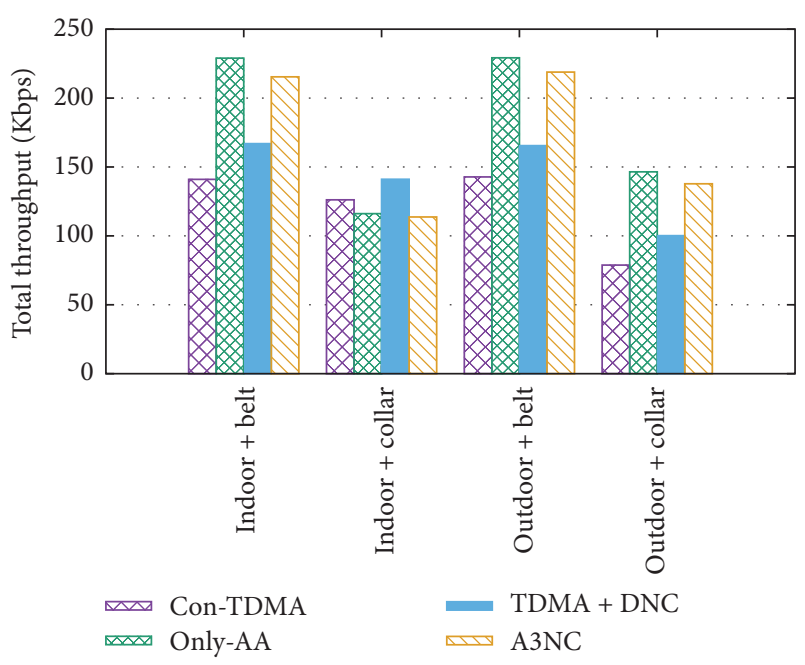

FIGURE 18: Throughputs of typical walking scenarios.

the belt, the PLD is relatively small when the hub is located on the back collar. Furthermore, the significant reflection effect in the indoor environment offsets the PLD between the two channels. Nevertheless, we find that Only-AA and A $3 \mathrm{NC}$ achieve a significant improvement in terms of upload throughput in the other three scenarios, which confirms the feasibility of AA method in the real walking scenarios.

Next, we explore relationship between the upload throughput and three key parameters: $\beta_{1}+\beta_{2}, \beta_{r}$, and $e$. Firstly, Figure 19 shows the results of total throughput versus $\beta_{1}+\beta_{2}$, when $\beta_{r}=0.9$ and the time shifting is $0 \mathrm{~ms}$. The Tx power of two sensors is amplified to increase the sum of $\beta_{1}$ and $\beta_{2}$; hence the value of $e$ is also augmented. The dash lines correspond to theoretical results derived from mathematical analyses in Section 5. The solid lines indicate the simulation results. Figure 19 clearly shows the good agreement between the mathematical analyses in Section 5 and the simulation results. The small deviation between the analytical and simulation curves is mainly due to two reasons. Firstly, the theoretical formulas analyze the throughput at the bit level, but the simulation model works at the packet level. The overhead of the packet header is not taken into account in the theoretical analyses. Meanwhile, because of the packetization of data, the wastage of the tail end of SUI (when the duration is too short to complete the transmission of one packet) is inevitable. Secondly, the idle interval between the transmission of two packets is not considered in the theoretical analysis.

Both theoretical and simulation results prove that Only$\mathrm{AA}$ and $\mathrm{A} 3 \mathrm{NC}$ achieve a remarkable improvement in comparison with Con-TDMA and TDMA-DNC, especially when $\beta_{1}+\beta_{2}=1$. Also, it can be seen the performances of Only$\mathrm{AA}, \mathrm{TDMA}+\mathrm{DNC}$, and A3NC plateau when the $\beta_{1}+\beta_{2}>1$. For the two schemes with AA mechanism, based on (10) and (16), the key part determining the throughput of Only-AA and $\mathrm{A} 3 \mathrm{NC}$ is $\beta_{1}+\beta_{2}-2 e$, that is, $\left(\beta_{1}-e\right)+\left(\beta_{1}-e\right)$. However, since the signal collisions increase with the amplifying of Tx power, $\beta_{1}-e$ and $\beta_{1}-e$ are limited under 0.5 . Therefore, after $\beta_{1}+\beta_{2}$ reaching one, further increase of $\beta_{1}$ or $\beta_{2}$ cannot

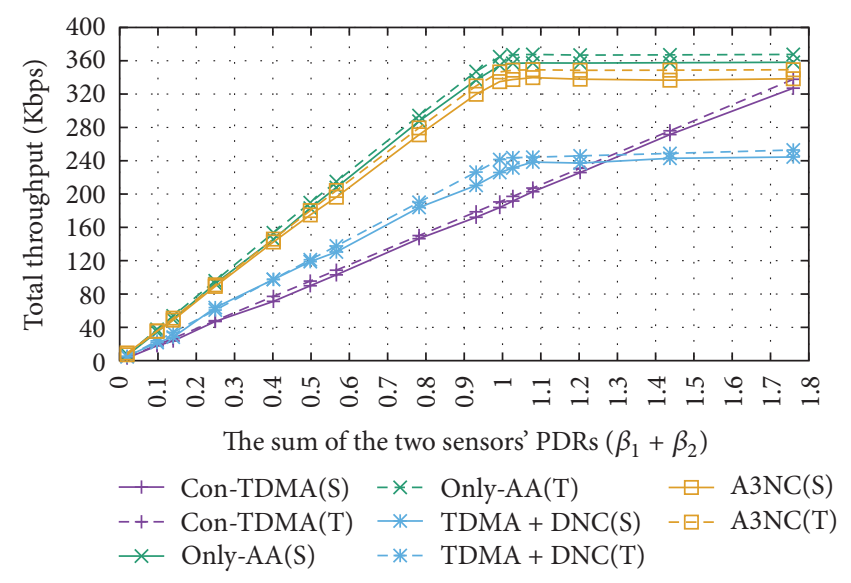

FIgURE 19: Throughput versus $\beta_{1}+\beta_{2}$.

improve the total throughput. For the TDMA + DNC scheme, based on the analysis result in (14), if $\beta_{r}=0.9$ and $\beta_{1}+$ $\beta_{2}>1$, the increment of $\beta_{1}$ or $\beta_{2}$ affects the throughput slightly. The reason is that, if the probability of successful relay cooperation is close to one, the reception of either one packet from the two sensors can lead to the decoding of the other packet. In other words, when $\beta_{1}+\beta_{2} \geq 1$, the hub can receive both two sensors' packets which is the best situation in one transmission process. Therefore, the upload throughput of the TDMA + DNC is saturated if $\beta_{1}+\beta_{2}>1$. As for the ConTDMA, the SUI of the two sensors are separated in the time domain and the relay node is not adopted, so the performance of Con-TDMA scheme raises linearly with the increase of $\beta_{1}+\beta_{2}$.

Figure 20 shows the throughput performance as a function of $\beta_{r}$, with the Tx power of $-38 \mathrm{dBm}$ and the time shifting of $0 \mathrm{~ms}$. As expected, the performances of both TDMA + $\mathrm{DNC}$ and $\mathrm{A} 3 \mathrm{NC}$ improve with the increase of $\beta_{r}$, while those of Only-AA and Con-TDMA remain constant. Besides, when the time shifting is 0 and Tx power is $-38 \mathrm{dBm}$ (i.e., $\beta_{1}+\beta_{2}<$ 1 ), $e$ is small enough to be ignored (around 0.022). Therefore, based on (14) and (16), the slope of TDMA + DNC is $1 / 3\left(1+\beta_{r}\right)$, while that slope of A3NC is $1 / 2\left(1+\beta_{r}\right)$. That is the reason why the curve of $\mathrm{A} 3 \mathrm{NC}$ is steeper than that of TDMA + DNC. In other words, $\mathrm{A} 3 \mathrm{NC}$ is more sensitive to the relay channel condition, which is acceptable when the relay node is deployed outside the human body.

In Figures 19 and 20, the time shifting is set to $0 \mathrm{~ms}$, which also matches the normal swing motion of arms in walking scenarios. However, if we increase the time shifting, the resulting change of $e$ will affect the system performance. Figure 21 shows the throughput performance as a function of $e$, when the other parameters are set to the default value (including $\beta_{1}+\beta_{2}=0.99$, and $\beta_{r}=0.9$ ). As expected, the performance of Con-TDMA is not affected by $e$, and the performances of the two schemes with AA mechanism decrease with the increase of $e$. Moreover, since $\beta_{r}=0.9$, the slope of two AA schemes are almost the same. An exception is that although AA mechanism is not adopted in TDMA + $\mathrm{DNC}$, its performance still declines with the rise of $e$, which 


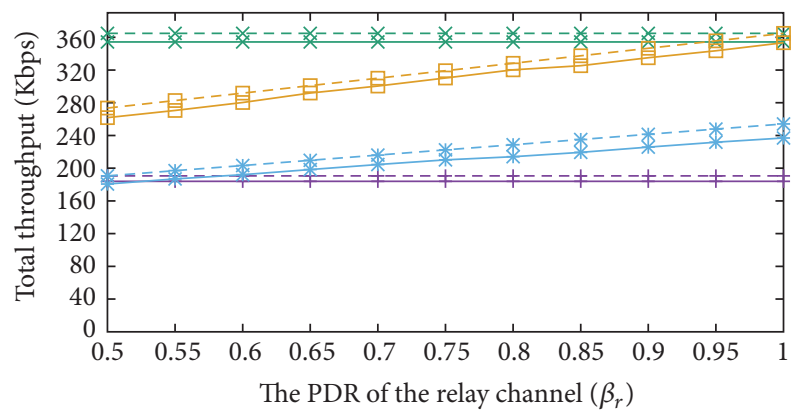

$$
\begin{aligned}
& \mp \text { Con-TDMA(S) }-*-\text { Only-AA(T) } \square \text { A3NC(S) } \\
& -+- \text { Con-TDMA(T) } \rightarrow \text { TDMA + DNC(S) }-\square-\text { A3NC(T) } \\
& * \text { Only-AA(S) } \quad-*-\mathrm{TDMA}+\mathrm{DNC}(\mathrm{T})
\end{aligned}
$$

FIgURE 20: Throughput versus $\beta_{r}$.

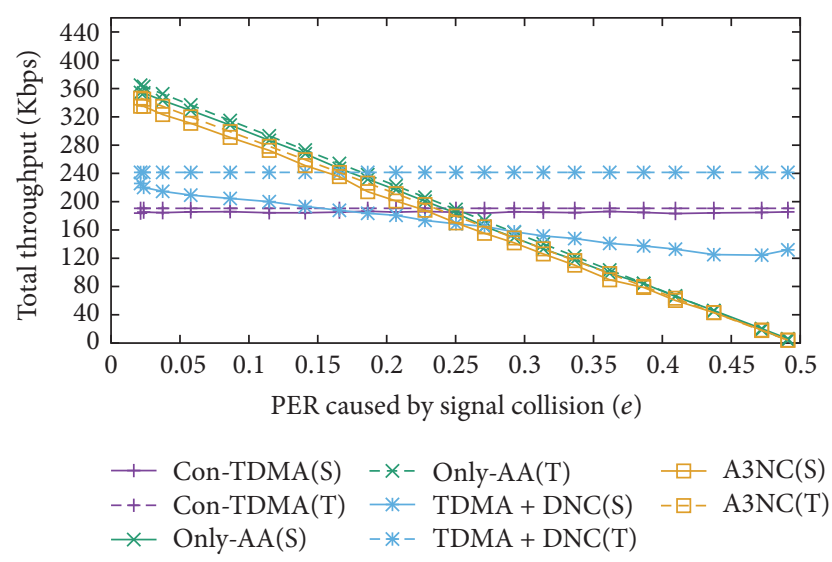

FIGURE 21: Throughput versus $e$.

does not match the analysis result in (14). The reason for this exception is that because the two SUI of the two sensors are adjacent and are relatively short compared to the walking cycle, when $e$ increases by changing the swing behavior of two arms, the hub tends to receive or discard both packets from the two sensors. Accordingly, the effectiveness of the relay cooperation is reduced.

6.2.2. Energy Efficiency. Figure 22 compares the energy efficiency of the four schemes when $\beta_{r}$ and time shifting are set to 0.9 and $0 \mathrm{~ms}$, respectively. Because both the PDR sum of the two sensors (i.e., $\beta_{1}+\beta_{2}$ ) and the energy consumption are affected by the Tx power of the sensors, we take the Tx power to evaluate the energy efficiency. The "energy efficiency" (EE) in Figure 22 represents how many megabytes (MB) of data are transmitted by consuming one joule energy of the two sensors (cf. (23)). As mentioned before, the energy consumption of the relay and hub is not considered in this paper.

From Figure 22, the energy efficiency of Only-AA, A3NC, and TDMA + DNC schemes increases slightly when the Tx power decreases from $-5 \mathrm{dBm}$ to $-38 \mathrm{dBm}$ and reduces rapidly when Tx power is below $-38 \mathrm{dBm}$. The curve trend can be explained by two reasons. First, combining the simulation results in Figures 19 and 16, when the Tx power is below

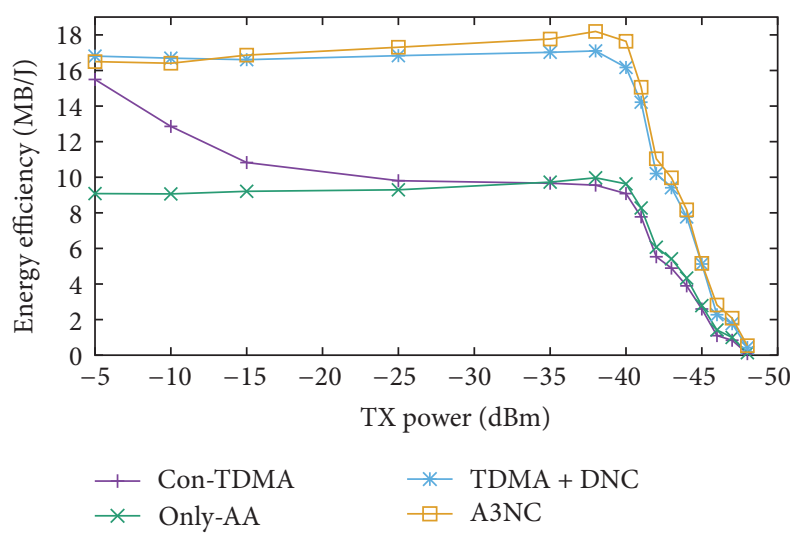

Figure 22: Measured energy efficiency (MByte/Joule) versus Tx power.

$-38 \mathrm{dBm}$ (i.e., $\beta_{1}+\beta_{1}<1$ ), the upload throughputs rise up with the increase of the Tx power, and the throughputs reach a plateau after Tx power $\geqslant 38 \mathrm{dBm}$. On the other hand, the working power of the sensor slightly reduces with the decline of TX power. Accordingly, based on the definition of EE in (23), the energy efficiency reaches the maximum at the point of $-38 \mathrm{dBm}$. As for the Con-TDMA scheme, since its throughput declines continuously with the decrease of $\mathrm{Tx}$ power (see Figure 19), its energy efficiency experiences a similar trend. At last, combining Figures 19 and 22, when the Tx power is $-38 \mathrm{dBm}$, both the throughput and the energy efficiency reach the maximum. The Tx power $-38 \mathrm{dBm}$ is considered as the optimal value for Only-AA, TDMA + DNC, and $\mathrm{A} 3 \mathrm{NC}$ for the simulation configuration.

We also estimate the lifetime for a typical WBAN application, in which an upload traffic of $200 \mathrm{kbps}$ is required for each sensor equipped with one CR2032 button battery. In this case, the TX powers of the two sensors are set to the optimal value: $-38 \mathrm{dBm}$. Figure 23 shows that the estimated lifetime of Con-TDMA or Only-AA is less than 12 days, while A3NC lasts over 21 days, which is a significant enhancement for WBANs systems.

6.2.3. Throughput Balance. All the above results are derived when the PDRs of two sensors are approximately equal, that is, $\beta_{1} \approx \beta_{2}$. Here we consider the case where $\beta_{1}$ and $\beta_{2}$ are different.

Figure 24 shows the average ratio between the upload throughput of the two sensors, $E\left(U_{2}\right) / E\left(U_{1}\right)$, as a function of $\beta_{2} / \beta_{1}$. The curves of both TDMA + DNC and A3NC are almost constant and are approximately equal to one, that is, $E\left(U_{1}\right) \approx E\left(U_{2}\right)$. That means the two schemes with relay cooperation can achieve a better throughput balance between the two sensors. In contrast, when the relay node is not utilized (Con-TDMA and Only-AA schemes), the difference between $E\left(U_{1}\right)$ and $E\left(U_{2}\right)$ increases significantly with the decrease of $\beta_{2} / \beta_{1}$. The simulation results in Figure 24 also confirm our analysis in Table 4 .

In summary, the simulation results confirm the theoretical analyses in Section 5. In contrast to conventional 


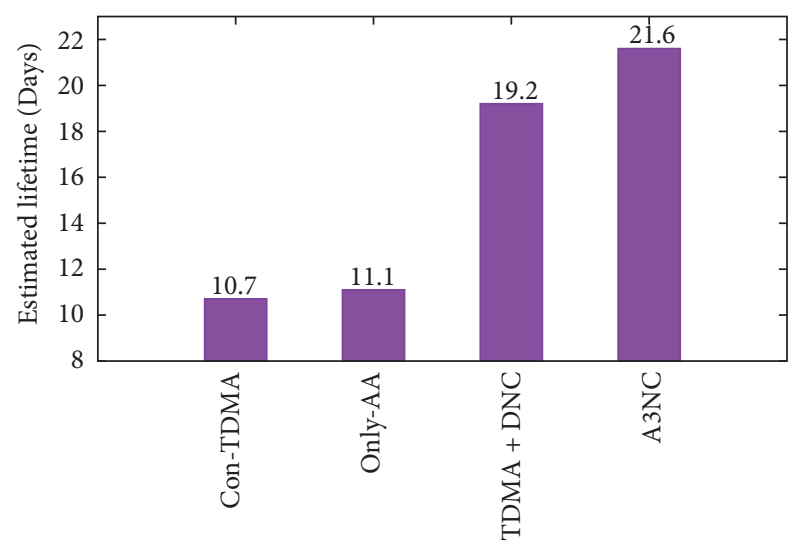

FIGURE 23: The comparison of the estimated lifetime.

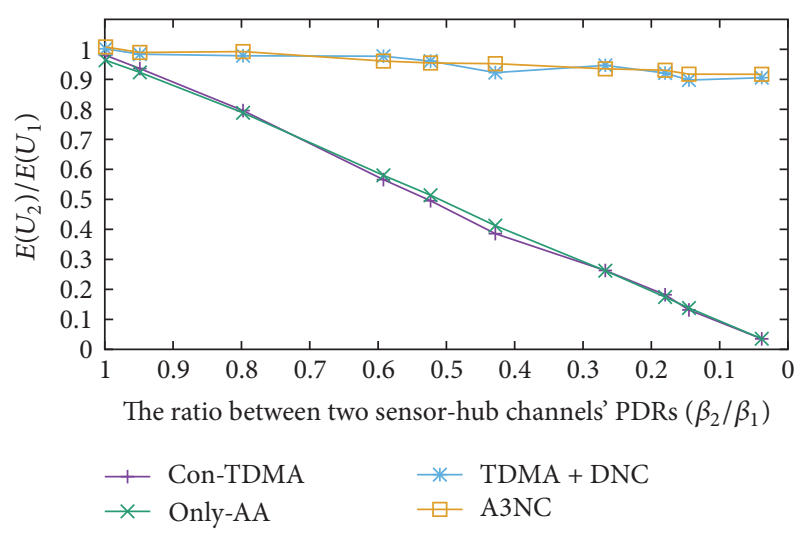

FIgURE 24: The comparison of throughput between the two sensors.

methods, the newly proposed A3NC scheme achieves a significant throughput improvement (about 10\%-40\% in normal walking scenarios), while attaining substantial power saving. Besides, with the help of cooperation communication, A3NC maintains a good throughput balance between two correlated sensor nodes.

\section{Conclusion}

In this paper, first, we introduced our customized portable wireless transceivers, which enable us to collect the realistic on-body wireless channel data in the walking scenarios. The statistical results of these collected datasets show that there exists a significant path loss discrepancy between two onbody channels when people are walking. This feature of onbody channel confirms the rationale of our newly proposed cross-layer network coding scheme, called A3NC. The A3NC scheme integrates the ANC technology in the PHY layer and a timeslot allocation algorithm in the MAC layer, to improve transmission performance in the walking scenarios. In $\mathrm{A} 3 \mathrm{NC}$, two sensors with significant path loss discrepancy simultaneously transmit packets to the relay and hub. Then, the relay forwards network coding packets to the hub and the hub performs the decoding process after the first two phases. We also derived the mathematical analyses for the proposed
A3NC from three perspectives, namely, sum throughput, energy efficiency, and throughput balance, in comparison with three other existing schemes. The validity of these performance analyses has been confirmed by our simulation. Simulation results show that the A3NC scheme achieves the best tradeoff between the energy efficiency and the throughput, compared to the other three existing schemes. Meanwhile, A3NC scheme provides a better sum throughput than the Con-TDMA and TDMA-DNC and roughly the same sum throughput as the Only-AA one. To the best of our knowledge, this is the first work attempting to bridge network coding to a spatially correlated WBAN system. For the future work, we plan to deepen the exploration of the on-body WBAN channels by considering more sensors and more generalized scenarios.

\section{Conflicts of Interest}

The authors declare that they have no conflicts of interest.

\section{References}

[1] S. Movassaghi, M. Abolhasan, J. Lipman, D. Smith, and A. Jamalipour, "Wireless body area networks: a survey," IEEE Communications Surveys and Tutorials, vol. 16, no. 3, pp. 1658-1686, 2014.

[2] "IEEE Standard for Local and metropolitan area networks - Part 15.6: Wireless Body Area Networks," IEEE Std. 802.15.6, 2012.

[3] K. Yekeh Yazdandoost and K. Sayrafian-Pour, "Channel model for body area network (BAN)," IEEE P802.15.6, 2009.

[4] J. N. Laneman, D. N. Tse, and G. W. Wornell, "Cooperative diversity in wireless networks: efficient protocols and outage behavior," IEEE Transactions on Information Theory, vol. 50, no. 12, pp. 3062-3080, 2004.

[5] L. C. Tran and A. Mertins, "Error performance and energy efficiency analyses of fully cooperative OFDM communication in frequency selective fading," IET Communications, vol. 10, no. 18, pp. 2525-2533, 2016.

[6] S. Ivanov, D. Botvich, and S. Balasubramaniam, "Cooperative wireless sensor environments supporting body area networks," IEEE Transactions on Consumer Electronics, vol. 58, no. 2, pp. 284-292, 2012.

[7] L. C. Tran, A. Mertins, X. Huang, and F. Safaei, "Comprehensive performance analysis of fully cooperative communication in WBANs," IEEE Access, vol. 4, pp. 8737-8756, 2016.

[8] Y. Chen, J. Teo, J. Lai et al., "Cooperative communications in ultra-wideband wireless body area networks: channel modeling and system diversity analysis," IEEE Journal on Selected Areas in Communications, vol. 27, no. 1, pp. 5-16, 2009.

[9] R. Ahlswede, N. Cai, S. R. Li, and R. W. Yeung, "Network information flow," IEEE Transactions on Information Theory, vol. 46, no. 4, pp. 1204-1216, 2000.

[10] S. Katti, H. Rahul, W. Hu, D. Katabi, M. Medard, and J. Crowcroft, "XORs in the air: practical wireless network coding," IEEE/ACM Transactions on Networking, vol. 16, no. 3, pp. 497510, 2008.

[11] D. S. Lun, M. Médard, and R. Koetter, "Efficient operation of wireless packet networks using network coding," in Proceedings of the International Workshop on Convergent Technologies (IWCT '05), Jun 2005. 
[12] G. E. Arrobo and R. D. Gitlin, "New approaches to reliable wireless body area networks," in Proceedings of the 2011 IEEE International Conference on Microwaves, Communications, Antennas and Electronic Systems (COMCAS '11), pp. 1-6, November 2011.

[13] G. E. Arrobo and R. D. Gitlin, "Improving the reliability of wireless body area networks," in Proceedings of the 33rd Annual International Conference of the IEEE Engineering in Medicine and Biology Society (EMBS '11), pp. 2192-2195, September 2011.

[14] P. M. P. Dharshini and M. Tamilarasi, "Adaptive reliable cooperative data transmission technique for wireless body area network," in Proceedings of the 2014 International Conference on Information Communication and Embedded Systems (ICICES '14), pp. 1-4, February 2014.

[15] E. Kartsakli, A. Antonopoulos, L. Alonso, and C. Verikoukis, "A cloud-assisted random linear network coding medium access control protocol for healthcare applications," Sensors, vol. 14, no. 3, pp. 4806-4830, 2014.

[16] Z. J. Haas and T.-C. Chen, "Cluster-based cooperative communication with network coding in wireless networks," in Proceedings of the 2010 IEEE Military Communications Conference (MILCOM '10), pp. 2082-2089, November 2010.

[17] X. Shi, M. Médard, and D. E. Lucani, "When both transmitting and receiving energies matter: an application of network coding in wireless body area networks," in Proceedings of the International Conference on Research in Networking, vol. 6827, pp. 119128, Springer, May 2011.

[18] S. Movassaghi, M. Shirvanimoghaddam, and M. Abolhasan, "A cooperative network coding approach to reliable wireless body area networks with demodulate-and-forward," in Proceedings of the 2013 9th International Wireless Communications and Mobile Computing Conference (IWCMC '13), pp. 394-399, July 2013.

[19] H. Alshaheen and H. T. Rizk, "Improving the energy efficiency for biosensor nodes in the WBSN bottleneck zone based on a random linear network coding," in Proceedings of the 2017 11th International Symposium on Medical Information and Communication Technology (ISMICT '17), pp. 59-63, Lisbon, Portugal, Feburary 2017.

[20] A. Taparugssanagorn, F. Ono, and R. Kohno, "Network coding for non-invasive wireless body area networks," in Proceedings of the 2010 IEEE 21st International Symposium on Personal, Indoor and Mobile Radio Communications Workshops (PIMRC '10), pp. 134-138, September 2010.

[21] M. A. Razzaque, S. S. Javadi, Y. Coulibaly, and M. T. Hira, "QoSaware error recovery in wireless body sensor networks using adaptive network coding," Sensors, vol. 15, no. 1, pp. 440-464, 2015.

[22] E. Byrne and A. Manada, "On coding schemes for wireless body area networks," in Proceedings of the 2012 International Symposium on Communications and Information Technologies (ISCIT '12), pp. 149-154, October 2012.

[23] S. Movassaghi, M. Shirvanimoghaddam, M. Abolhasan, and D. Smith, "An energy efficient network coding approach for wireless body area networks," in Proceedings of the 38th Annual IEEE Conference on Local Computer Networks (LCN '13), pp. 468-475, October 2013.

[24] E. Kartsakli, A. Antonopoulos, A. Lalos et al., "Reliable MAC design for ambient assisted living: Moving the coordination to the cloud," IEEE Communications Magazine, vol. 53, no. 1, pp. 78-86, 2015.

[25] A. S. Lalos, A. Antonopoulos, E. Kartsakli et al., "RLNC-aided cooperative compressed sensing for energy efficient vital signal telemonitoring," IEEE Transactions on Wireless Communications, vol. 14, no. 7, pp. 3685-3699, 2015.

[26] R. D'Errico and L. Ouvry, "Time-variant BAN channel characterization," in Proceedings of the 2009 IEEE 20th Personal, Indoor and Mobile Radio Communications Symposium (PIMRC '09), pp. 3000-3004, September 2009.

[27] H. Feng, B. Liu, Z. Yan, C. Zhang, and C. W. Chen, "Predictionbased dynamic relay transmission scheme for wireless body area networks," in Proceedings of the 2013 IEEE 24th Annual International Symposium on Personal, Indoor, and Mobile Radio Communications (PIMRC '13), pp. 2539-2544, September 2013.

[28] H. Zhang, F. Safaei, and L. C. Tran, "Joint analog network coding and channel allocation in the walking scenario for WBAN," in Proceedings of the 2016 IEEE Symposium on Computers and Communication (ISCC '16), pp. 604-609, July 2016.

[29] H. Zhang, F. Safaei, and L. C. Tran, "Measurement-based characterizations of on-body channel in the human walking scenario," 2017, http://www.uow.edu.au/ lctran/publications_ files/VTC2017spring.pdf.

[30] L. Hanlen, V. Chaganti, B. Gilbert, D. Rodda, T. Lamahewa, and D. Smith, "Open-source testbed for body area networks: 200 sample/sec, $12 \mathrm{hrs}$ continuous measurement," in Proceedings of the 2010 IEEE 21st International Symposium on Personal, Indoor and Mobile Radio Communications Workshops (PIMRC '10), pp. 66-71, September 2010.

[31] M. Lauzier, P. Ferrand, A. Fraboulet, H. Parvery, and J.M. Gorce, "Full mesh channel measurements on Body Area Networks under walking scenarios," in Proceedings of the 2013 7th European Conference on Antennas and Propagation (EuCAP '13), pp. 3508-3512, April 2013.

[32] K. Pearson, "Note on regression and inheritance in the case of two parents," Proceedings of the Royal Society of London, vol. 58, pp. 240-242, 1895.

[33] J. G. Proakis and M. Salehi, Digital Communications, McGrawHill, 5th edition, 2007.

[34] J. G. Proakis and M. Salehi, Fundamentals of Communication Systems, Pearson Education, 2007.

[35] S. Katti, S. Gollakota, and D. Katabi, "Embracing wireless interference: analog network coding," in Proceedings of the 2007 conference on Applications, technologies, architectures, and protocols for computer communications (SIGCOMM '07), vol. 37, no. 2, pp. 397-408, Kyoto, Japan, August 2007.

[36] A. Boulis, "Castalia: a simulator for wireless sensor networks and body area networks," [Online], https://github.com/ boulis/Castalia/. 


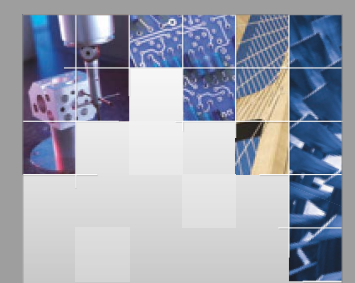

\section{Enfincering}
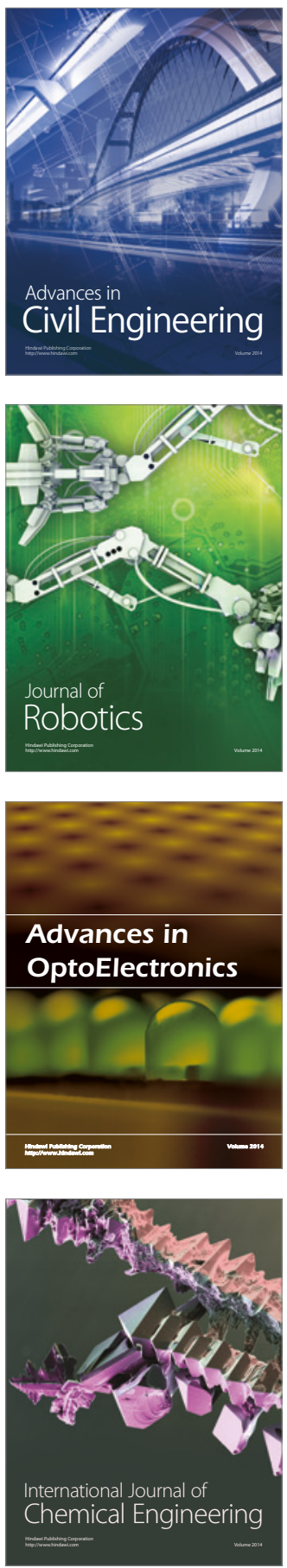

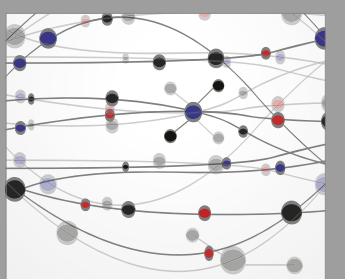

The Scientific World Journal

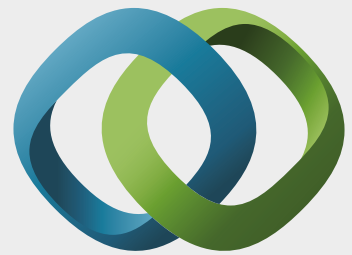

\section{Hindawi}

Submit your manuscripts at

https://www.hindawi.com
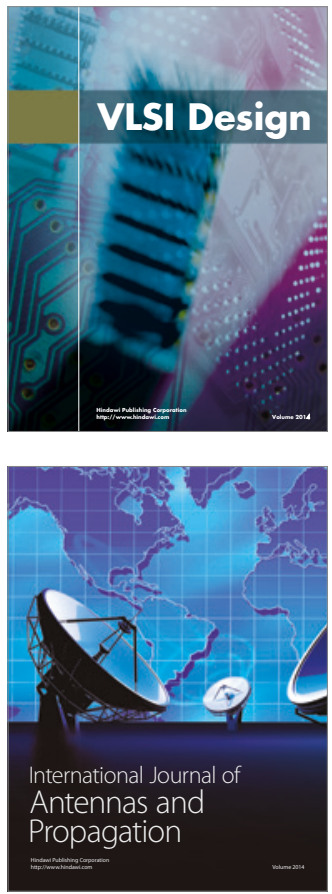

\section{Rotating}

Machinery
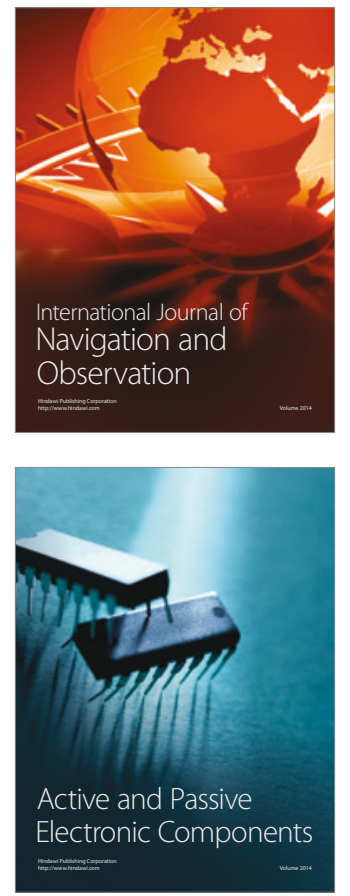
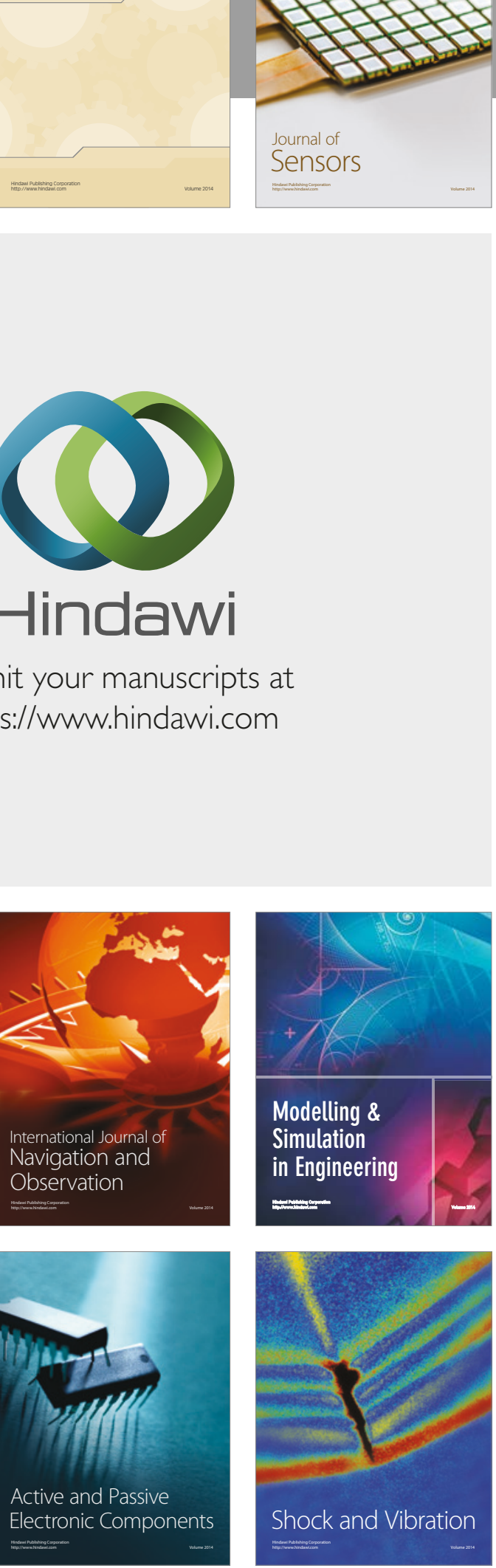
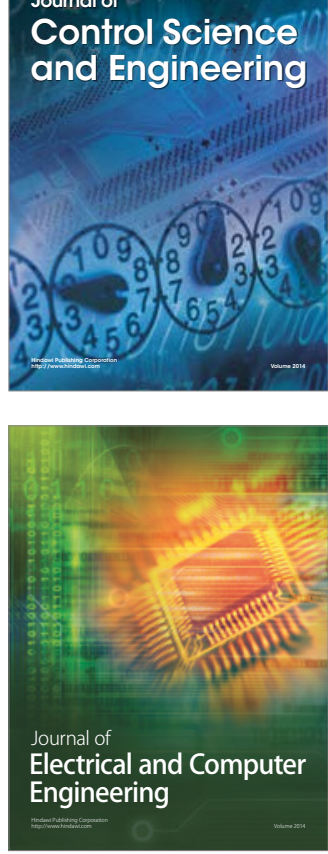

Distributed

Journal of

Control Science

and Engineering
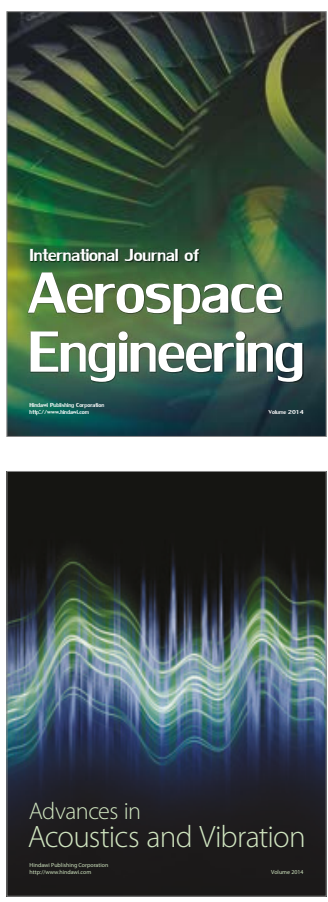

Sensor Networks 